\title{
Exact-WKB, complete resurgent structure, and mixed anomaly in quantum mechanics on $S^{1}$
}

\author{
Naohisa Sueishi, ${ }^{a}$ Syo Kamata, ${ }^{b}$ Tatsuhiro Misumi ${ }^{c, d}$ and Mithat Ünsal ${ }^{e}$ \\ ${ }^{a}$ Department of Physics, Nagoya University, \\ Nagoya 464-8602, Japan \\ ${ }^{b}$ National Centre for Nuclear Research, \\ 02-093 Warsaw, Poland \\ ${ }^{c}$ Department of Mathematical Science, Akita University, \\ Akita 010-8502, Japan \\ ${ }^{d}$ Department of Physics, Keio University, \\ Kanagawa 223-8521, Japan \\ ${ }^{e}$ Department of Physics, North Carolina State University, \\ Raleigh, NC 27607, U.S.A. \\ E-mail: sueishi@eken.phys.nagoya-u.ac.jp, skamata11phys@gmail.com, \\ tatsuhiromisumi@gmail.com, unsal.mithat@gmail.com
}

ABSTRACT: We investigate the exact-WKB analysis for quantum mechanics in a periodic potential, with $N$ minima on $S^{1}$. We describe the Stokes graphs of a general potential problem as a network of Airy-type or degenerate Weber-type building blocks, and provide a dictionary between the two. The two formulations are equivalent, but with their own pros and cons. Exact-WKB produces the quantization condition consistent with the known conjectures and mixed anomaly. The quantization condition for the case of $N$-minima on the circle factorizes over the Hilbert sub-spaces labeled by discrete theta angle (or Bloch momenta), and is consistent with 't Hooft anomaly for even $N$ and global inconsistency for odd $N$. By using Delabaere-Dillinger-Pham formula, we prove that the resurgent structure is closed in these Hilbert subspaces, built on discrete theta vacua, and by a transformation, this implies that fixed topological sectors (columns of resurgence triangle) are also closed under resurgence.

KEYwords: Discrete Symmetries, Nonperturbative Effects, Resummation, Solitons Monopoles and Instantons

ArXiv EPrint: 2103.06586 


\section{Contents}

1 Introduction 1

1.1 Three related theories 2

1.2 Review of exact-WKB and general strategy 4

$2 S^{\mathbf{1}}$ quantum mechanical system with Airy-type Stokes graphs 5

2.1 Quantization condition 5

$\begin{array}{ll}2.2 & \text { Gutzwiller trace formula } \\ & 10\end{array}$

2.3 For $V(x)=1-\cos (N x) \quad 11$

3 Hilbert space perspective and $\mathbb{Z}_{N}$ gauging $\quad 13$

3.1 Factorization of exact quantization condition from Hilbert space perspective 13

$\begin{array}{lll}3.2 & \text { Factorization of exact quantization from path integral description } & 15\end{array}$

4 Analysis of the degenerate Weber-type Stokes graphs 17

4.1 Relation between Airy-type and degenerate Weber-type Stokes graphs 17

$\begin{array}{lll}4.2 & \text { From quantization condition to partition function } & 18\end{array}$

4.3 Resurgent structure of the Hilbert space and the partition function 23

5 Summary and discussion $\quad 26$

A The degenerate Weber equation $\quad 28$

$\begin{array}{lll}\text { A.1 Derivation of the connection formula } & 28\end{array}$

A.2 Construction of the dictionary 34

$\begin{array}{lll}\text { A.2.1 } A \text {-cycle } & 34\end{array}$

$\begin{array}{lll}\text { A.2.2 } B \text {-cycle } & 35\end{array}$

\section{Introduction}

Recently, the application of resurgence theory and exact-WKB analysis to quantum theory has been attracting a great deal of attention. The main statement of the resurgence theory in quantum theory is that the perturbative and non-perturbative contributions have a nontrivial relation and one can understand many aspects of non-perturbative physics just from the perturbative series. The resurgence theory has been intensively investigated in terms of mathematics [1], quantum mechanics [2-21], matrix models and string theory [22-34] and quantum field theory [35-43]. The resurgent structure and the related Stokes phenomena are understood by two different methods including semi-classical analysis and the exactWKB analysis [44-68]. In our previous work [64], we obtained the unified understanding of the two Stokes phenomena in semi-classical description of path integral and exact-WKB analyses. The Stokes phenomenon leading to the ambiguous contribution by the structure of Lefschetz thimble for the quasi-zero mode direction for instanton-antiinstanton critical 
point at infinity corresponds to the change of the "topology" of the Stoke curves in the exact-WKB analysis. We also found the relation between Maslov index and the intersection number of Lefschetz thimble. The results we obtained in [64] is summarized in the flowchart figure 1.

In this paper, we study quantum mechanics of a particle on $S^{1}$ in the presence of periodic potential. We consider $N$-minima on the circle where $N=1,2, \ldots$. The application of the exact-WKB analysis to these systems is of great importance in terms of understanding resurgent structure in theories with topological $\theta$ angle, discrete 't Hooft anomaly [69], quantization conditions [7, 13], the Mathieu equation [53] and TBA equations $[56,59,60,65]$. Furthermore, these QM systems provide a simpler prototype for circle compactified $\mathbb{C P}^{N-1}$ on $\mathbb{R} \times S^{1}[35,38,40]$ and deformed Yang-Mills theory on $\mathbb{R}^{3} \times S^{1}[70,71]$. We show that the quantization condition for the system with $N$ minima on $S^{1}$ factorizes according to the $N$-Bloch momenta (or equivalently, $N$ discrete $\theta$ angles), corresponding to decomposition of Hilbert space $\mathcal{H}=\bigoplus_{p=0}^{N-1} \mathcal{H}_{p}$. By obtaining the exact partition function of the system based on the exact-WKB analysis, we show that the resurgent structure is closed in each $\mathcal{H}_{p}$, the eigenspace of the shift operator. This implies that, by a Fourier transform, the topological sectors $Q \in \mathbb{Z}$ which correspond to columns of resurgence triangle, are also closed under Stokes automorphisms and resurgence. We also show that quantization condition for $N$ even model at $\theta=\pi$ becomes a perfect square, corresponding spectral doubling, and produce the mixed 't Hooft anomaly between $\mathbb{Z}_{N}$ translation symmetry and $C$ charge conjugation symmetry [69].

We elucidate the Stokes graphs of the system. For a classical potential problem, we describe how Stokes graphs can be expressed as a network of Airy type or degenerate Weber type building blocks. We show how the perturbative and non-perturbative cycles are related in terms of the resurgent structures. The resultant quantization condition is in exact agreement to the conjectured one by Zinn-Justin-Jentschura [7] and DunneUnsal [13]. We also exhibit the dictionary to connect the cycles of the Airy-type $\left(E_{0} \neq 0\right)$ and degenerate Weber-type $\left(E_{0}=0\right)$ Stokes graphs, where the latter is more suitable for merging pair of turning points.

This paper is constructed as follows: in section 1.2, we review the exact WKB analysis, with emphasis on its relation to the resurgence theory and the known quantization conditions, based on our previous work. In section 2, we study the quantum mechanical systems on $S^{1}$ (periodic-potential systems) by the exact-WKB method with the Airy-type Stokes graph, and obtain the conjectured quantization condition. In section 3, we introduce the Hilbert-space perspective and discuss the gauging of $\mathbb{Z}_{N}$ symmetry, with emphasis on its relation to TQFT. In section 4 , we study the $S^{1}$ quantum mechanical systems by the degenerate Weber-type Stokes graph instead of the Airy type, and obtain the quantization condition without any approximation, leading to the partition function with the exact resurgent structure. Section 5 is devoted to the summary and the discussion.

\subsection{Three related theories}

There are few quantum mechanical systems whose local dynamics are identical, but global structure and Hilbert space structures are different. These can be related to each other in 


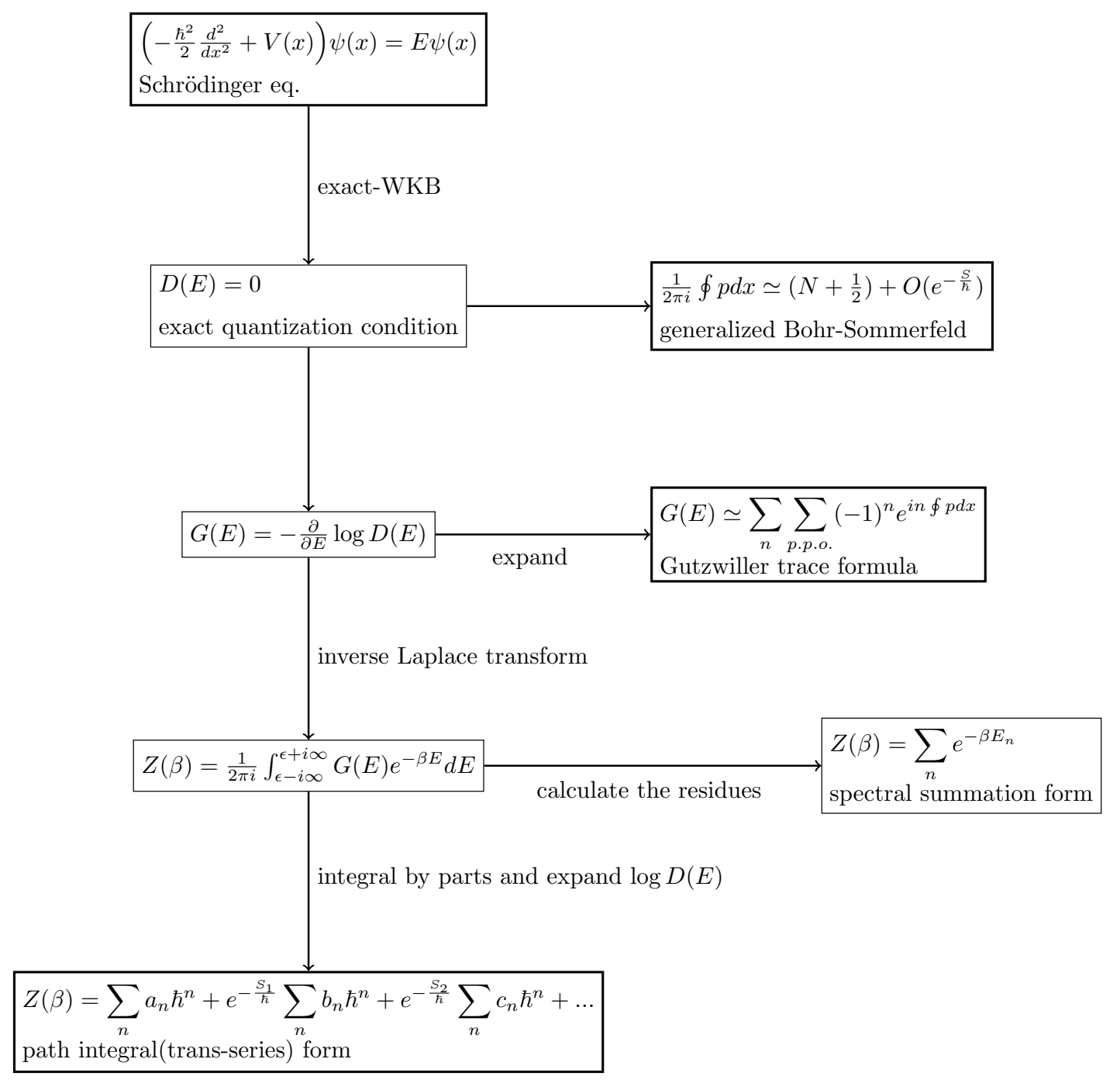

Figure 1. The relation among several quantization methods $\left(\mathbb{Z}_{N}\right.$-shift symmetry given by $x \rightarrow$ $x+2 p \pi / N$.). We can identify the resurgent structure of each case without approximation from the exact-WKB.

a precise way, and our analysis, with some modifications, obviously apply to all three. For clarity, we briefly describe these three systems and their salient features.

- Particle on a line $x \in \mathbb{R}$ in the presence of a periodic potential $V(x+2 \pi)=V(x)$. This system has a $\mathbb{Z}$ translation symmetry. Hilbert space is composed of the bands and each band has infinitely many states labelled by Bloch momenta $k a \in[-\pi, \pi]$ (we set lattice spacing $a=1$ in general.) In this construction, there is no theta angle.

- Gauging $\mathbb{Z}$ translation symmetry completely, we end up with particle on a circle, $x \in S^{1}=\mathbb{R} / 2 \pi \mathbb{Z}$. Now, $x \sim x+2 \pi$ are physically identified (due to gauging), and there is only one minimum of the potential in the fundamental domain, $x \in S^{1}$. In this system, translation is no longer a global symmetry, it is fully gauged. Only one state from each band of particle on an infinite line $\mathbb{R}$ is present in the Hilbert space. 
One can add a theta angle to this system. Theta angle determines which Bloch state of the energy band survives in the Hilbert space upon gauging, with identification $k a \equiv \theta$. We can call the Hilbert space based on this theta vacuum as $\mathcal{H}_{\theta}$. In the exact WKB analysis, we generally use this set-up.

- Gauging $N \mathbb{Z}$ subgroup of $\mathbb{Z}$ translation symmetry, we end up with particle on a circle, $x \in S^{1}=\mathbb{R} / 2 \pi N \mathbb{Z}$. Now, $x \sim x+2 \pi N$ are physically identified and there are $N$ perturbative minima of the potential in the fundamental domain $x \in S^{1}$. This system has a genuine global $\mathbb{Z}_{N}$ translation symmetry. Now, $N$ states from each band are present in the Hilbert space, and these are labelled by $N$ distinct discrete Bloch momenta (also called discrete theta angle in this context). One can add a continuous theta angle to this system as well. We use this system in exact WKB analysis to probe mixed anomalies. We call this system $T_{N}$ model for brevity. This set-up can be used to extrapolate between $N=1$ particle on $S^{1}$ case and particle on an infinite line $x \in \mathbb{R}$.

These systems possess exactly the same local dynamics. Their perturbation theories, instanton and bion data are completely equivalent. But their Hilbert spaces and global symmetries are distinct. As emphasized, the first system has infinitely many states per band, the second system has one state per band, and the third system has $N$ states per band. Yet, one can obtain the whole spectral data of the first system from second and third. In the exact WKB analysis, we first use the second setup to derive quantization condition for a particular theta angle, and build Hilbert space on top of a certain theta vacuum $|\theta\rangle, \mathcal{H}_{\theta}$. We also prove that quantization condition that produces $\operatorname{Spec}\left[\mathcal{H}_{\theta}\right]$ is invariant under Stokes automorphism, and DDP formula. By a Fourier transform, this shows that traditional resurgence (relating late terms with early terms) is also closed on the fixed topological charge sectors, which are the columns of resurgence triangle [13]. Finally, we use the third set-up to demonstrate the emergence of mixed anomalies at $\theta=\pi$ for even $N$.

\subsection{Review of exact-WKB and general strategy}

We first briefly review the exact-WKB analysis and its relation to resurgence theory, see [64] for details. One of the most important advantages of the exact-WKB analysis is that we obtain the quantization condition from the normalization condition of the wavefunctions in $x \rightarrow \pm \infty$ limits of the Stokes graph. The quantization condition is regarded as the zero condition of the Fredholm determinant as

$$
D(E)=\operatorname{det}(\widehat{H}-E)=0,
$$

where $D(E)$ denotes the Fredholm determinant. It enables us to derive exact energy eigenvalues in principle. Furthermore, once we obtain the Fredholm determinant, we also have the resolvent $G(E)$ and the partition function $Z(\beta)$ straightforwardly as

$$
\begin{aligned}
& G(E)=\operatorname{tr} \frac{1}{\widehat{H}-E}=-\frac{\partial}{\partial E} \log D(E), \\
& Z(\beta)=\operatorname{tr} e^{-\beta \widehat{H}}=\frac{1}{2 \pi i} \int_{\epsilon-i \infty}^{\epsilon+i \infty} G(E) e^{-\beta E} d E .
\end{aligned}
$$


In the previous work [64], we make use of these facts and show that the Stokes phenomena in the semiclassical path-integral analysis (bion analysis [72-81]) are realized as the global alternation of the Stokes graph in the exact-WKB analysis, where the perturbative and nonperturbative contributions correspond to the different cycles crossing the Stokes curves.

In the exact-WKB analysis, the Stokes phenomenon and the related resurgent structure between the perturbative and nonperturbative contributions are determined by the Stokes curve and its associated monodromy matrix. It is notable that the Stokes curve is uniquely determined by the lowest order of the WKB expansion, i.e., the classical potential.

Earlier work [64] also brought an understanding of the relation between the exact-WKB analysis and the other known quantization methods. In particular, the trace of resolvent $G(E)$ gives the Gutzwiller trace formula [82],

$$
G(E)=\operatorname{tr} \frac{1}{\widehat{H}-E}=i \sum_{p . p . o .} \sum_{n=1}^{\infty} T(E) e^{i n \oint_{\text {p.p.o. }} p d x}(-1)^{n}\left(\left|\operatorname{det} \frac{\delta^{2} S}{\delta x \delta x}\right|\right)^{-1 / 2} .
$$

This form is interpreted as the intermediate quantization method between the path integral and the Bohr-Sommerfeld quantizations. This method gives the resolvent of the system by summing up periodic classical solutions.

These facts are summarized in the flowchart shown in figure 1. It is important to note that, since the Fredholm determinant $D(E)$ obtained by the exact-WKB analysis is exact, what follows from there, e.g. the trace of resolvent $G(E)$ and the partition function $Z(\beta)$ are also exact.

\section{$2 \quad S^{1}$ quantum mechanical system with Airy-type Stokes graphs}

\subsection{Quantization condition}

We here discuss the exact-WKB analysis for the particle on a circle, $x \in S^{1}$, where $x \sim$ $x+2 \pi$ are physically identified, in the presence of the potential, $V(x)=1-\cos (x)$. Since the target space is $S^{1}$, we can turn on topological $\theta$ angle, which correspond to the insertion of the Aharanov-Bohm flux through the circle. Our main purpose is to derive the quantization condition from the periodicity condition of the system and WKBwave function $\psi(x+2 \pi)=e^{-i \theta} \psi(x)$. In the sequential subsections, we will obtain the Gutzwiller trace formula of this system, then we will extend our analysis to the cases with $V(x)=1-\cos (N x)$, corresponding to $N$-minima in the fundamental domain.

We begin with Schrödinger equation

$$
\left(-\frac{\hbar^{2}}{2} \frac{d^{2}}{d x^{2}}+V(x)\right) \psi(x)=E \psi(x) .
$$

Set $Q(x)=2(V(x)-E)$, rewrite the equation as

$$
\left(-\frac{d^{2}}{d x^{2}}+\hbar^{-2} Q(x)\right) \psi(x)=0
$$


In the WKB analysis, we consider the ansatz given by

$$
\psi(x, \hbar)=e^{\int^{x} S(x, \hbar) d x},
$$

which leads to the to the non-linear Riccati equation

$$
S(x)^{2}+\frac{\partial S}{\partial x}=\hbar^{-2} Q(x)
$$

Next, we assume that $S(x, \hbar)$ has a formal power series expansion in expansion parameter $\hbar$,

$$
S(x, \hbar)=\hbar^{-1} S_{-1}(x)+S_{0}(x)+\hbar S_{1}(x)+\hbar^{2} S_{2}(x)+\ldots,
$$

where $S_{n}(x)$ are functions of $x$. This leads to recursive equation

$$
S_{-1}^{2}=Q(x), \quad 2 S_{-1} S_{n}+\sum_{j=0}^{n-1} S_{j} S_{n-j}+\frac{\partial S_{n-1}}{\partial x}=0 \quad(n \geq 0) .
$$

Since $S_{n}$ is recursively determined from $S_{-1}= \pm \sqrt{Q}, S_{n}$ has two independent solutions:

$$
\begin{aligned}
S^{ \pm}(x, \hbar) & =\hbar^{-1} S_{-1}^{ \pm}(x)+S_{0}^{ \pm}(x)+\hbar S_{1}^{ \pm}(x)+\hbar^{2} S_{2}^{ \pm}(x)+\ldots \\
& = \pm \hbar S_{-1}^{+}+S_{0}^{+} \pm \hbar S_{1}^{+}+\hbar^{2} S_{2}^{+}+\ldots \\
& = \pm S_{\text {odd }}+S_{\text {even }} .
\end{aligned}
$$

Then the WKB wave functions can be expressed as

$$
\psi_{a}^{ \pm}(x)=e^{\int^{x} S^{ \pm} d x}=\frac{1}{\sqrt{S_{\text {odd }}}} e^{ \pm \int_{a}^{x} S_{\text {odd }} d x},
$$

with $a$ being an integral constant. For later calculations, we choose it as a turning point, which is a solution of $Q(x)=0$.

Since we have derived the WKB wave function recursively, it is regarded as a formal series in $\hbar$

$$
\begin{aligned}
\psi_{a}^{ \pm}(x) & =e^{ \pm \frac{1}{\hbar} \int_{a}^{x} \sqrt{Q(x)} d x} \sum_{n=0}^{\infty} \psi_{a, n}^{ \pm}(x) \hbar^{n+\frac{1}{2}} \\
S_{\text {odd }} & =\sum_{n=0}^{\infty} S_{2 n-1} \hbar^{2 n-1}
\end{aligned}
$$

Here, both of these series turn out to be asymptotic expansions with respect to $\hbar$. The exact-WKB analysis considers the Borel summation of each series and their Stokes phenomena [64].

From now on, we focus on the periodic potential $V(x)=1-\cos (x)$, and $x \in S^{1}$. We now determine the Stokes curve, which dictates where the Stokes phenomenon of WKB wave function occurs. Let $a$ be a turning point (a solution of $Q(x)=0$ ). The Stokes curve associated with $a$ is defined as

$$
\operatorname{Im} \frac{1}{\hbar} \int_{a}^{x} \sqrt{Q(x)} d x=0 .
$$



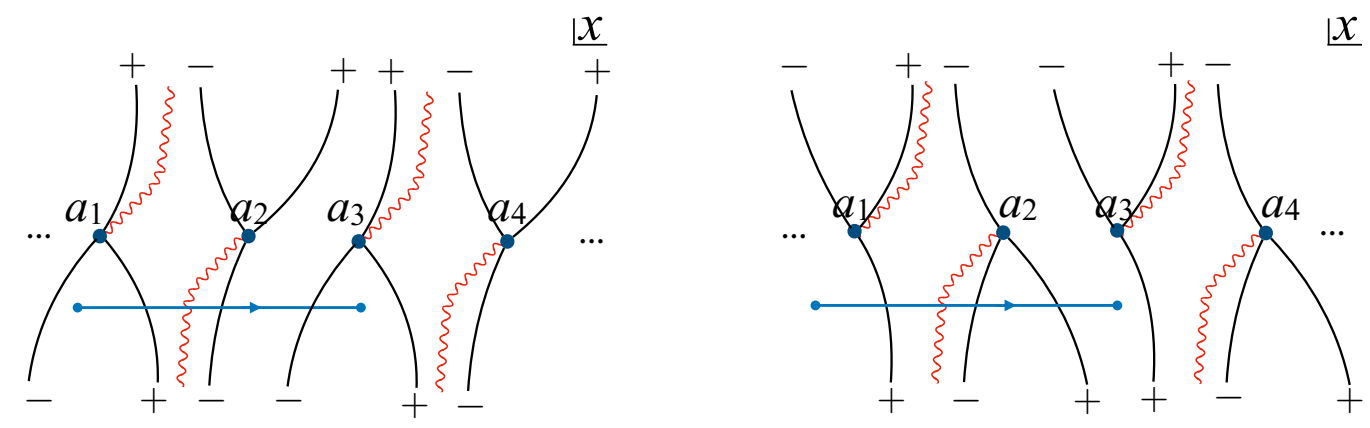

Figure 2. The Stokes curve for the two periods of the potential $1-\cos (x)$ for $\operatorname{Im}(\hbar)>0$ and $\operatorname{Im}(\hbar)<$ 0 , respectively. We also depict the branch cut, the turning points and the path corresponding to the single period.
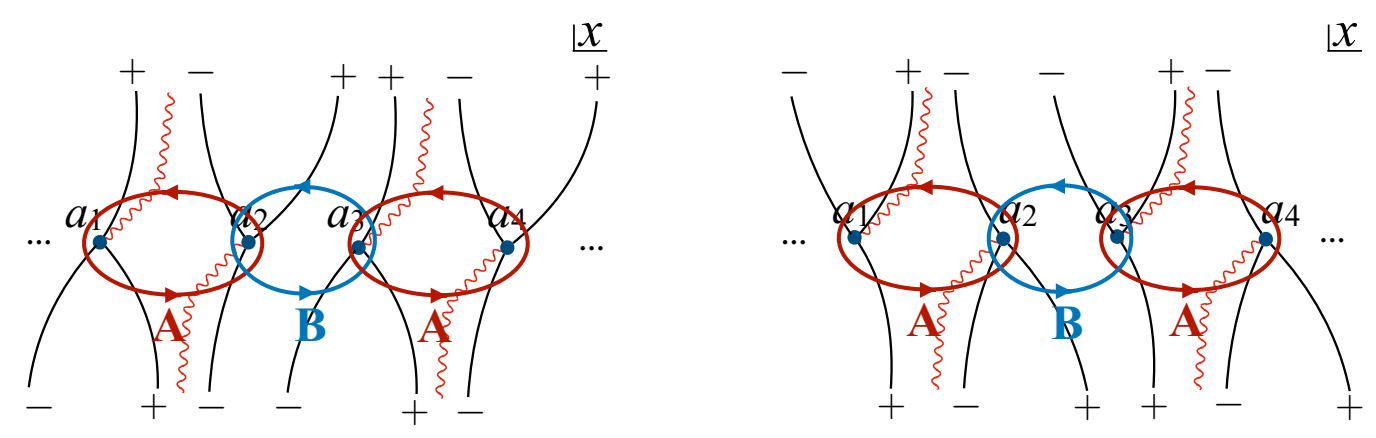

Figure 3. The Stokes curve for the two periods of the potential $1-\cos (x)$, with the cycles $A$ and $B$ being depicted.

Each segment of the Stokes curve has an index, \pm . This index indicates which one of the $\psi^{+}$and $\psi^{-}$pair increases exponentially when moving from the point $a$ to infinity along the Stokes curve. When the index of the corresponding Stokes curve is,$+ \psi^{+}$increases exponentially with

$$
\operatorname{Re} \frac{1}{\hbar} \int_{a}^{x} \sqrt{Q(x)} d x>0
$$

When the index is - , then $\psi^{-}$increases exponentially with

$$
\operatorname{Re} \frac{1}{\hbar} \int_{a}^{x} \sqrt{Q(x)} d x<0 .
$$

In the present case, the Stokes curve is depicted in figures 2 and 3. In figure 2 we depict Stokes curves for the two periods of the potential, exhibiting four turning points $a_{1}, a_{2}, a_{3}, a_{4}$ and the path corresponding to a single period by a blue line. In figure 3 we also exhibit the perturbative cycle $A=e^{\oint_{A} S_{\text {odd }}}=e^{2 \int_{a_{1}}^{a_{2}} S_{\text {odd }}}=e^{2 \int_{a_{3}}^{a_{4}} S_{\text {odd }}}$, and the nonperturbative cycle $B=e^{\oint_{A} S_{\text {odd }}}=e^{2 \int_{a_{2}}^{a_{3}} S_{\text {odd }}}$, in the same figure. The nonperturbative cycle $B$ corresponds to the single bion contribution $\propto e^{-S_{\text {bion }} / \hbar}$. We note that, although this Stokes curve is specific to the potential $V(x)=1-\cos (x)$, any periodic potential has one with the same topological property. 
Based on the Stokes graph, we derive the quantization condition below for the present potential. We here consider the single-period path depicted in figure 3 . What we have to do is just to find out the monodromy matrices appearing when the path is crossing the Stokes curves. As we cross the Stokes graph through the full period, the monodromy matrices for $\operatorname{Im} \hbar>0$ are given as

$$
\begin{aligned}
\left(\begin{array}{l}
\psi_{a_{1}}^{+}(x) \\
\psi_{a_{1}}^{-}(x)
\end{array}\right) & =M_{+} T N_{a_{1} a_{2}} M_{-} N_{a_{2} a_{3}} M_{-}\left(\begin{array}{l}
\psi_{a_{3}=a_{1}}^{+}(x+2 \pi) \\
\psi_{a_{3}=a_{1}}^{-}(x+2 \pi)
\end{array}\right) \\
& \equiv \mathcal{M}^{+}\left(\begin{array}{c}
\psi_{a_{1}}^{+}(x+2 \pi) \\
\psi_{a_{1}}^{-}(x+2 \pi)
\end{array}\right),
\end{aligned}
$$

and these for $\operatorname{Im} \hbar<0$ are given as

$$
\begin{aligned}
\left(\begin{array}{l}
\psi_{a_{1}}^{+}(x) \\
\psi_{a_{1}}^{-}(x)
\end{array}\right) & =M_{+} T N_{a_{1} a_{2}} M_{-} M_{+} N_{a_{2} a_{3}}\left(\begin{array}{l}
\psi_{a_{3}=a_{1}}^{+}(x+2 \pi) \\
\psi_{a_{3}=a_{1}}^{-}(x+2 \pi)
\end{array}\right) \\
& \equiv \mathcal{M}^{-}\left(\begin{array}{c}
\psi_{a_{1}}^{+}(x+2 \pi) \\
\psi_{a_{1}}^{-}(x+2 \pi)
\end{array}\right) .
\end{aligned}
$$

Here, $M_{ \pm}$acts while passing \pm labelled Stokes line in the counter-clockwise direction, $T$ acts on the crossing of the branch cut in the counter-clock-wise direction, $N_{a_{1} a_{2}}$ is the Voros multiplier accounting the change of turning points entering the WKB wave-function. These are explicitly given by:

$$
\begin{aligned}
M_{+} & :=\left(\begin{array}{cc}
1 & i \\
0 & 1
\end{array}\right), & M_{-}:=\left(\begin{array}{ll}
1 & 0 \\
i & 1
\end{array}\right) \\
T & :=\left(\begin{array}{cc}
0 & -i \\
-i & 0
\end{array}\right), & N_{a_{1} a_{2}}:=\left(\begin{array}{cc}
e^{+\int_{a_{1}}^{a_{2}} S_{\text {odd }}} & 0 \\
& e^{-\int_{a_{1}}^{a_{2}} S_{\text {odd }}}
\end{array}\right) .
\end{aligned}
$$

We now impose a boundary condition on the above wave-function specific to the periodic potential. Because of the $2 \pi$ periodicity of $x$, the wave-function must satisfy $\psi(x+2 \pi)=e^{-i \theta} \psi(x)$. We then have the condition

$$
\mathcal{M}^{ \pm}\left(\begin{array}{l}
\psi_{a_{1}}^{+}(x) \\
\psi_{a_{1}}^{-}(x)
\end{array}\right)=e^{i \theta}\left(\begin{array}{l}
\psi_{a_{1}}^{+}(x) \\
\psi_{a_{1}}^{-}(x)
\end{array}\right)
$$

This is nothing but the eigenvalue equation of $\mathcal{M}^{ \pm}$. Therefore, we obtain

$$
\operatorname{det}\left(\mathcal{M}^{ \pm}-e^{i \theta} I\right)=0,
$$

where $I$ is a $2 \times 2$ unit matrix. This result means that the Fredholm determinant $D(E)$ in the quantization condition $D(E)=0$ is $D^{ \pm}=\frac{1}{e^{i \theta}} \operatorname{det}\left(\mathcal{M}^{ \pm}-e^{i \theta} I\right)$, where \pm indicates the sign of imaginary term of $\hbar$. We now write down the quantization condition for the present periodic potential as ${ }^{1}$

$$
D^{ \pm}(E)=\frac{1}{\sqrt{A^{\mp 1} B}}\left(1+A^{\mp 1}+A^{\mp 1} B-2 \sqrt{A^{\mp 1}} \sqrt{B} \cos \theta\right)=0 .
$$

\footnotetext{
${ }^{1}$ The overall constant doesn't affect the quantization condition and chosen for simplicity.
} 
This result agrees with [53] where quantization condition for Mathieu equation was obtained by use of the exact-WKB method as well. For our purpose, (2.21) is a building block, as it will become manifest in our treatment of a potential with $N$-minima instead of one in the fundamental domain, as discussed in section 2.3. This generalization will allow us to make a precise link between exact WKB method and mixed 't Hooft anomalies. Furthermore, we will use (2.21) to prove that fixed topological charge sectors of the theory (corresponding to the columns of resurgence triangle) are closed under Stokes automorphism.

The resurgent structure of $D^{ \pm}(E)$ is determined by Delabaere-Dillinger-Pham (DDP) formula $[44,52]$.

$$
\mathcal{S}_{+}[\sqrt{A}]=\mathcal{S}_{-}[\sqrt{A}](1+\mathcal{S}[B])
$$

where $\mathcal{S}_{ \pm}$is directional/lateral Borel summation ${ }^{2}$ for $\operatorname{sign}(\operatorname{Im} \hbar)= \pm 1$. ( $B$ cycle does not have Borel singularity so $\mathcal{S}_{+}[B]=\mathcal{S}_{-}[B]=\mathcal{S}[B]$.) The DDP formula states that the left/right Borel resummation of the perturbative $A$-cycle is dictated by the Borel resummation of the non-perturbative $B$ cycle. Using DDP formula, we can show that left/right Borel resummation of the exact quantization condition are equal:

$$
\mathcal{S}_{+}\left[D^{+}\right]=\mathcal{S}_{-}\left[D^{-}\right]
$$

Therefore, the Fredholm determinant is invariant under the change of directions (left or right) in the Borel summation or equivalently, under Stokes automorphism.

We can show a physical meaning of this condition. To show the non-perturbative contribution to the ground state energy, we consider the asymptotic form of $A$, which does not include non-perturbative contribution before being Borel-resummed. It is written as

$$
A \rightarrow e^{-2 \pi i \frac{E}{\hbar \omega_{A}(E, \hbar)}}
$$

where $\omega_{A}(E, \hbar)$ is an asymptotic expansion with respect to $\hbar$. In the low-energy limit, it is regarded as a harmonic frequency of the classical vacuum as

$$
\begin{aligned}
& \omega_{A}(E, \hbar)^{2}=\sum_{n=0}^{\infty} c_{n}(E) \hbar^{n} \\
& \lim _{E \rightarrow 0} c_{0}(E)=V^{\prime \prime}\left(x_{\mathrm{vac}}\right),
\end{aligned}
$$

where $x_{\text {vac }}$ is a minimum of the potential. This expression corresponds to taking the Borel-resummed $A$ back to its asymptotic expansion form. We now express the energy eigenvalues as $E=\hbar \omega_{A}\left(\frac{1}{2}+\delta\right)$. Then, the non-perturbative energy deviation $\delta$ from the harmonic oscillator is

$$
\sin (\pi \delta)= \pm i \frac{1}{2} B e^{ \pm \pi i \delta}-\sqrt{B} \cos \theta
$$

\footnotetext{
${ }^{2}$ The Borel summation is a homomorphism, so that the following algebraic properties hold: $\mathcal{S}[A+B]=$ $\mathcal{S}[A]+\mathcal{S}[B], \mathcal{S}[A B]=\mathcal{S}[A] \mathcal{S}[B]$.
} 
and it is approximated as

$$
\delta \sim-\frac{1}{\pi} \sqrt{B} \cos \theta \pm i \frac{1}{2 \pi} B
$$

We note that $\sqrt{B}$ corresponds to the instanton contribution $\propto e^{-\left(S_{\text {bion }} / 2\right) / \hbar}=e^{-S_{I} / \hbar}$, with $S_{I}$ being the instanton action. One finds that this expression of the ground state energy indicates the instanton contribution shifts the energy by an amount dictated by $\theta$ angle, while the bion contribution leads to the imaginary ambiguity associated with the Stokes phenomena. The ambiguity cancels against the Borel resummation of perturbation theory. The meaning of (2.23) is that this type of resurgent cancellation takes place to all nonperturbative orders.

Despite the elegance of the Airy-type analysis of exact-WKB, this formalism is not always most suitable, especially when the turning points merge. This limit requires some extra work to get the spectral information correctly, and this task does not seem to be very insightful. Instead, we discuss the method of degenerate Weber-type exact-WKB. The formalism we already built-in for Airy will be quite useful there, and we will also provide a dictionary between these two types of exact-WKB. Weber-type exact-WKB produce spectral data correctly as discussed in section 4 .

\subsection{Gutzwiller trace formula}

The Gutzwiller trace formula is a semiclassical construction that express the quantum mechanical density of states (the resolvent, $G(E)$ ), in terms of periodic orbits, which is called prime periodic orbit (p.p.o.). It is generally difficult to determine what the p.p.o. are and how they are added. But this data can be easily extracted by using the quantization condition obtained by exact-WKB, as shown in [64]. In this subsection, we show the structure of Gutzwiller trace formula of $S^{1}$ system and how Stokes phenomenon corresponding to the imaginary term of $\hbar$ appears in this formalism.

$$
\begin{aligned}
D^{ \pm} & \propto 1+A^{\mp 1}+A^{\mp} B-2(\sqrt{A})^{\mp 1} \sqrt{B} \cos \theta \\
& =\left(1+A^{\mp 1}\right)\left(1+\frac{B}{1+A^{ \pm 1}}-\frac{\sqrt{B}}{\sqrt{A}+\frac{1}{\sqrt{A}}}\left(e^{i \theta}+e^{-i \theta}\right)\right)
\end{aligned}
$$

Using $G^{ \pm}(E)=-\frac{\partial}{\partial E} \log D^{ \pm}$

$$
\begin{aligned}
G_{\mathrm{pt}}^{ \pm}(E) & =-\left(\frac{\partial}{\partial E} A^{\mp 1}\right) \sum_{n=0}^{\infty}(-1)^{n} A^{\mp n} \\
G_{\mathrm{np}}^{ \pm}(E) & =-\left(\frac{\partial}{\partial E} K\right) \sum_{m=0}^{\infty}(-1)^{m} K^{m} \\
K & =B \sum_{n=0}^{\infty}(-1)^{n} A^{ \pm n}-\sqrt{B} \sum_{n=0}^{\infty}(-1)^{n} A^{ \pm\left(n+\frac{1}{2}\right)}\left(e^{i \theta}+e^{-i \theta}\right)
\end{aligned}
$$

Now $A=e^{\oint_{A} S_{\text {odd }} d x} \simeq e^{\frac{i}{\hbar} \oint_{A}|p| d x}$ and $B \simeq e^{-\frac{1}{\hbar} \oint_{B}|p| d x}$. From (2.30), we can identify the Gutzwiller trace formula and p.p.o., which includes the non-perturbative contribution 

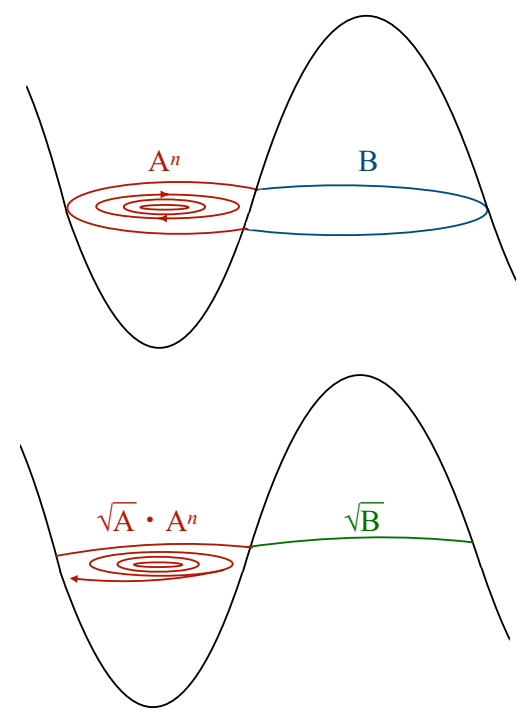

Figure 4. To be periodic, $B A^{n}$ and $\sqrt{B} A^{n+\frac{1}{2}}$ is the unit of nonperturbative contribution. The Stokes phenomenon corresponding to the bion ambiguity is regarded as $A \rightarrow A^{-1}$.

in a periodic system. The derivative term $\frac{\partial}{\partial E} A=\frac{1}{\hbar}\left(\oint_{A} \frac{1}{\sqrt{2(V(x)-E)}} d x+O(\hbar)\right) A=\frac{i}{\hbar} T_{A} A$ gives the period. ${ }^{3}$ The $(-1)^{n}$ associated with each periodic orbit is the Maslov index. ${ }^{4}$ The physical meaning of the form of $K$, which is the unit of the non-perturbative contribution, can also be understood by considering its orbit. There are two kinds of fundamental nonperturbative periodic orbits, $B A^{n}$ and $\sqrt{B} A^{n+\frac{1}{2}}$ in the periodic potential as shown in figure 3. Actually, the infinite number of $A$ cycle attached to $B$ or $\sqrt{B}$ in the expression of $K$ is regarded as quasi-moduli integral in terms of the path integral method [64] and we show it explicitly in 4.2 .

\subsection{For $V(x)=1-\cos (N x)$}

For more generic cases $V(x)=1-\cos (N x)(N \in \mathbb{N}$.), we can also obtain the Fredholm determinant and the quantization condition. The monodromy matrix unit $\mathcal{M}^{ \pm}$is diagonalized as

$$
U^{-1} \mathcal{M}^{ \pm} U=\left(\begin{array}{cc}
\xi-\sqrt{\xi^{2}-1} & 0 \\
0 & \xi+\sqrt{\xi^{2}-1}
\end{array}\right)=\left(\begin{array}{ll}
\alpha & 0 \\
0 & \beta
\end{array}\right)
$$

with $\xi=\frac{1}{2 \sqrt{A^{ \pm 1} B}}\left(1+A^{ \pm 1}+B\right)$. Here $\alpha, \beta$ are the roots of $x^{2}-2 \xi x+1=0$, where we have $\alpha+\beta=2 \xi, \alpha \beta=1$. We then have the quantization condition as

$$
\begin{aligned}
\operatorname{det}\left(\left(\mathcal{M}^{ \pm}\right)^{N}-e^{i \theta} I\right) & =\operatorname{det}\left(\left(U^{-1} \mathcal{M}^{ \pm} U\right)^{N}-e^{i \theta} I\right) \\
& =e^{i \theta}\left(2 \cos \theta-\alpha^{N}-\beta^{N}\right)=0
\end{aligned}
$$

\footnotetext{
${ }^{3}$ This period includes quantum fluctuation $O(\hbar)$. The original Gutzwiller trace formula is derived with semi-classical approximation, so such the fluctuation term (including the higher order in $A, B$ ) gives the correction for the Gutzwiller trace formula.

${ }^{4}$ More precisely, the Maslov index is $\alpha$, where $(-1)^{n}=e^{i \alpha \pi}$.
} 
which is rewritten as

$$
\begin{aligned}
D^{ \pm}(E) & =\alpha^{N}+\beta^{N}-2 \cos \theta \\
& =\left\{2 \sum_{\ell=0}^{\left\lfloor\frac{N}{2}\right\rfloor}\left(\begin{array}{c}
N \\
N-2 \ell
\end{array}\right)\left(\frac{1+A^{ \pm}+B}{2 \sqrt{A^{ \pm} B}}\right)^{N-2 \ell}\left(\frac{\left(1+A^{ \pm}+B\right)^{2}}{4 A^{ \pm} B}-1\right)^{\ell}\right\}^{2}-2 \cos \theta \\
& =\frac{1}{\left(A^{\mp 1} B\right)^{N / 2}} \prod_{p=0}^{N-1}\left[1+A^{\mp 1}(1+B)-2 \sqrt{A^{\mp 1} B} \cos \left(\frac{\theta+2 \pi p}{N}\right)\right] \\
& \equiv \prod_{p=0}^{N-1} D_{p}^{ \pm}(E)
\end{aligned}
$$

where $\lfloor x\rfloor$ is the floor function and $\left(\begin{array}{c}N \\ N-2 \ell\end{array}\right)$ is the binomial coefficient. This is one of the most important results in this paper. First, it shows that the exact quantization condition factorizes to N-building blocks. These building blocks are labelled by a Bloch momentum (discrete theta angle). This factorization is due to the fact that Hilbert space decompose to eigenstates of $\mathbb{Z}_{N}$ translation operator, and we explain the details of this in the next section.

When $N=2 K \quad(K \in \mathbb{N})$ and $\theta=\pi$, the exact quantization condition (2.33) becomes a perfect square:

$$
\begin{aligned}
D(E) & =\left(\alpha^{K}+\beta^{K}\right)^{2} \\
& =\frac{1}{\left(A^{\mp 1} B\right)^{K}} \prod_{p=0}^{K-1}\left[1+A^{\mp 1}(1+B)-2 \sqrt{A^{\mp 1} B} \cos \left(\frac{\pi(2 p+1)}{2 K}\right)\right]^{2} .
\end{aligned}
$$

This indicates that all the energy eigenvalues are doubly degenerate (Kramers doubling). This degeneracy is regarded as a result of 't Hooft anomaly between $\mathbb{Z}_{N}$ discrete translation symmetry and $C=\mathbb{Z}_{2}$ charge conjugation symmetry. The existence of 't Hooft anomaly means that a trivial gap with a unique ground state is prohibited. Thus, the ground state should be degenerate if a mixed 't Hooft anomaly exists of the quantum mechanical models. In the next section, we will discuss the gauging of $\mathbb{Z}_{N}$ symmetry, and the result of the mixed 't Hooft anomaly in detail.

When $N=2 K+1 \quad(K \in \mathbb{N})$ and $\theta=0, \pi$, the exact quantization condition (2.33) becomes

$$
\begin{aligned}
& D(E, \theta=0)=D_{p=0}(E, \theta=0) \prod_{p=1}^{K}\left[D_{p}(E, \theta=0)\right]^{2} \\
& D(E, \theta=\pi)=D_{p=K}(E, \theta=\pi) \prod_{p=0}^{K-1}\left[D_{p}(E, \theta=\pi)\right]^{2} .
\end{aligned}
$$

There are $K$ pairs, and a singlet sector. The sector that is not paired up at $\theta=0$ and $\theta=\pi$ are distinct, and they are not continuously connected. This is the global inconsistency condition [84]. It is slightly milder condition than mixed anomaly, but essentially plays similar role. Exact quantization naturally captures global inconsistency condition as well. 


\section{$3 \quad$ Hilbert space perspective and $\mathbb{Z}_{N}$ gauging}

In this section we introduce the Hilbert-space perspective and interpret our result of the quantization condition in eq. (2.33). We also discuss the gauging of $\mathbb{Z}_{N}$ symmetry and show that the mixed 't Hooft anomaly is encoded in our result in eq. (2.33).

\subsection{Factorization of exact quantization condition from Hilbert space perspec- tive}

Consider quantum mechanics of a particle on a circle with the potential

$$
V(x)=1-\cos (N x), \quad x \sim x+2 \pi
$$

For brevity, we call it $T_{N}$ model [83]. This theory has a discrete $\mathbb{Z}_{N}$ translation symmetry,

$$
\mathbb{Z}_{N}: x \mapsto x+\frac{2 \pi}{N}
$$

whose generator we denote with $\mathrm{U}$. Since $[H, \mathrm{U}]=0$, eigenstates of Hamiltonian are also eigenstates of discrete translation operator. Denote eigenstates as $|n, p\rangle$ where $n$ is band label and $p$ is the label of the Bloch momentum associated with $\mathbb{Z}_{N}$ symmetry, obeying

$$
H|n, p\rangle=E_{n, p}|n, p\rangle, \quad \mathrm{U}|n, p\rangle=e^{i 2 \pi p / N}|n, p\rangle
$$

The $\mathrm{U}$ operator obeys $\mathbb{Z}_{N}$ group multiplication law:

$$
\mathrm{U}^{\ell_{1}} \mathrm{U}^{\ell_{2}}=\mathrm{U}^{\ell_{1}+\ell_{2} \bmod N}
$$

Given the $\mathrm{U}$ operator, we can built projection operator to Bloch momentum $p$ states that is useful to decompose the Hilbert space into Bloch sectors:

$$
\Pi_{p}=\frac{1}{N} \sum_{\ell=0}^{N-1} \omega^{\ell p} \mathrm{U}^{\ell}
$$

The projection operators satisfy the standard relations:

$$
\sum_{p=0}^{N-1} \Pi_{p}=\mathbf{1}, \quad \Pi_{p}^{2}=\Pi_{p}, \quad \Pi_{p_{1}} \Pi_{p_{2}}=0 \text { if } p_{1} \neq p_{2} \bmod N
$$

and can be used to decompose the Hilbert space of the theory into sectors

$$
\mathcal{H}=\bigoplus_{p=0}^{N-1} \mathcal{H}_{p}
$$

according to Bloch momenta. This decomposition is one reason for the factorization of the exact quantization condition (2.33). Relatedly, $\mathcal{H}_{p}$ subspaces in this decomposition will emerge naturally as we gauge $\mathbb{Z}_{N}$ symmetry, as the full Hilbert space of $\left(T_{N} / \mathbb{Z}_{N}\right)_{p}$ models. 


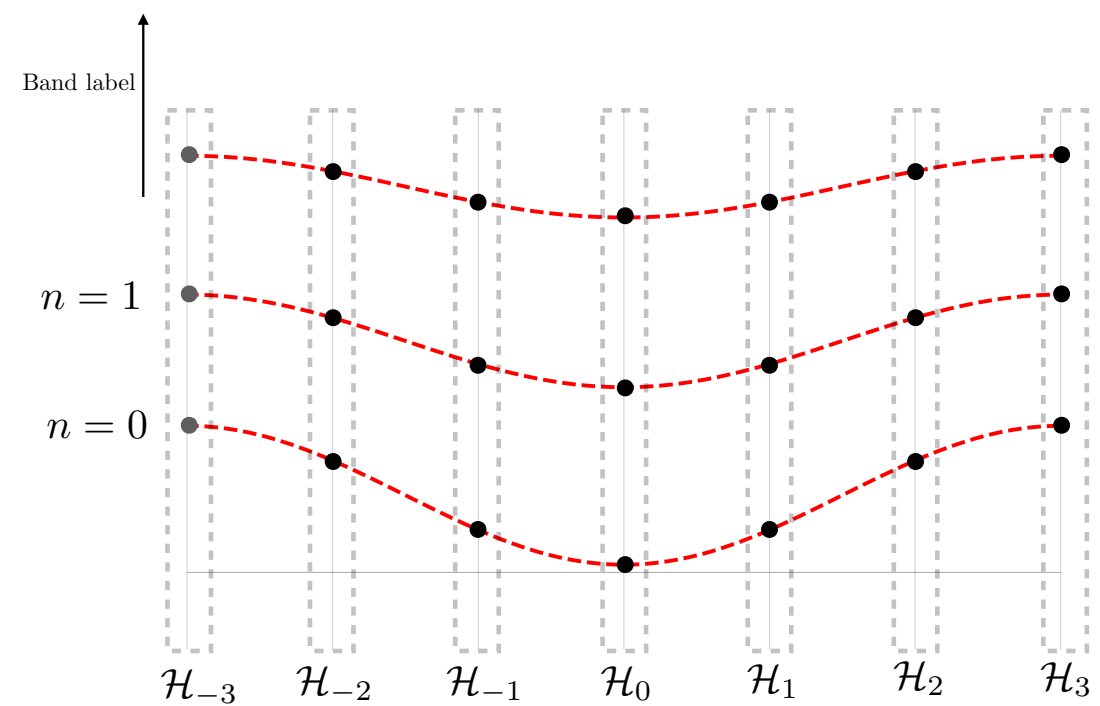

Figure 5. The Hilbert space of the original $T_{N}$ model can be decomposed according to $\mathbb{Z}_{N}$ quantum numbers associated with Bloch momenta $p . \theta_{p}=\frac{2 \pi p}{N}$ can be viewed as a discrete theta angle, or the coefficient of Chern-Simons term in topological gauge theory. $\mathcal{H}_{p}$ acquires an interpretation as Hilbert space of $\left(T_{N} / \mathbb{Z}_{N}\right)_{p}$ theory. The exact quantization conditions of the $T_{N}$ theory factorizes into the quantization conditions for the $\left(T_{N} / \mathbb{Z}_{N}\right)_{p}$ models.

We can see the implication of the (3.7) in the partition function. The partition function of the $T_{N}$ model can be written as

$$
Z_{0}(\beta)=\operatorname{tr}\left[e^{-\beta H}\right]=\sum_{p=0}^{N-1}\left(\sum_{n} e^{-\beta E_{n, p}}\right) \equiv \sum_{p=0}^{N-1} \widetilde{Z}_{p}(\beta)
$$

where $\widetilde{Z}_{p}(\beta)$ is the partition function of the sub-system with fixed Bloch momentum $p$. Let us also define the partition function with the insertion of translation operator,

$$
Z_{\ell}(\beta)=\operatorname{tr}\left[e^{-\beta H} \mathrm{U}^{\ell}\right]=\sum_{p=0}^{N-1} e^{i 2 \pi p \ell / N} \tilde{Z}_{p}(\beta)
$$

This is just regular partition function for $\ell=0 . Z_{\ell}(\beta)$ and $\widetilde{Z}_{p}(\beta)$ are related via a discrete Fourier transformation.

Now, we can describe in an elementary way gauging of $\mathbb{Z}_{N}$ symmetry, see $[69,84]$. Physically, gauging translation symmetry is the declaration that $x$ and $x+\frac{2 \pi}{N}$ are physically equivalent points. So, the size of the $S^{1}$ circle is reduced from $2 \pi$ down to $\frac{2 \pi}{N}$. Hence, there is only one minimum on the fundamental domain of gauged $T_{N} / \mathbb{Z}_{N}$ theory. This means, on each band on the Hilbert space, instead of $N$ states, only one state survives, i.e. each band is diluted by a factor of $N$.

More formal description of gauging is as follows. Global symmetry generators are a set of codim- 1 defects, (point defects in the present case). We can gauge the discrete global symmetries by summing over all possible networks of such codim-1 defects, namely, 
summing over $\ell$,

$$
\widetilde{Z}_{0}(\beta) \equiv Z_{\left(T_{N} / \mathbb{Z}_{N}\right)_{0}}=\operatorname{tr}\left[e^{-\beta H} \Pi_{0}\right] \equiv \frac{1}{N} \sum_{\ell=0}^{N-1} Z_{\ell}
$$

The projection to zero Bloch momentum states guarantees this partition function corresponds to the Hilbert space $\mathcal{H}_{0}$ in the Bloch decomposition (3.7). However, this is not the only possibility for gauging. The gauging procedure admits the freedom to add a topological phase, a discrete topological theta angle, $\theta_{p}=\frac{2 \pi p}{N}$ to each network configuration of the topological defects. This is equivalent to the insertion of other projection operators (3.5) into the state sum. Hence,

$$
\widetilde{Z}_{p}(\beta) \equiv Z_{\left(T_{N} / \mathbb{Z}_{N}\right)_{p}}=\operatorname{tr}\left[e^{-\beta H} \Pi_{p}\right] \equiv \frac{1}{N} \sum_{\ell=0}^{N-1} e^{-\mathrm{i} \frac{2 \pi \ell p}{N}} Z_{\ell}
$$

Using the fact that $Z_{\ell}=\sum_{n} \sum_{k=0}^{N-1} e^{\mathrm{i} \frac{2 \pi \ell k}{N}} e^{-\beta E_{n, k}}$ where $n$ is band and $k$ is Bloch momentum label, we can immediately deduce that the sum reduce to

$$
\widetilde{Z}_{p}(\beta) \equiv Z_{\left(T_{N} / \mathbb{Z}_{N}\right)_{p}}=\sum_{|n, p\rangle \in \mathcal{H}_{p}} e^{-\beta E_{n, p}}
$$

This is just the set of states in the Hilbert space $\mathcal{H}_{p}$ in the decomposition (3.7).

As a result of decomposition of Hilbert space according to discrete theta angle $\theta_{p}$, the quantization condition in the $T_{N}$ model with topological theta angle $\theta$ and the one in the $\left(T_{N} / \mathbb{Z}_{N}\right)_{p}$ theories must be related by the factorization formula:

$$
D_{T_{N}}(E)=\prod_{p=0}^{N-1} D_{\left(T_{N} / \mathbb{Z}_{N}\right)_{p}}(E)
$$

in exact agreement with the formula (2.33) obtained from exact WKB analysis.

\subsection{Factorization of exact quantization from path integral description}

There is also some benefit to be gained to present this construction in path integral, especially, for the identification of Bloch momentum (which acts as a label in the decomposition of Hilbert space), which in turn is a discrete theta angle. The origin of this term is a topological Chern-Simons term in quantum mechanics $[85,86]$. This discussion is slightly more abstract compared to our explicit Hilbert space construction, but it generalize more naturally to QFT. For this reason, we provide a short over-view of the path integral formulation of coupling of QM to $\mathbb{Z}_{N}$ topological gauge theory.

Since we will ultimately gauge the $\mathbb{Z}_{N}$ global symmetry (3.2) in our quantum mechanical system, it is first useful to describe $\mathbb{Z}_{N}$ topological gauge theory. The $\mathbb{Z}_{N}$ gauge field can be described by a pair of fields $\left(A^{(1)}, A^{(0)}\right)$, which obeys

$$
N A^{(1)}=\mathrm{d} A^{(0)}, \quad \int A^{(1)}=\frac{1}{N} \int \mathrm{d} A^{(0)}=\frac{2 \pi}{N} \ell, \quad \ell \in \mathbb{Z}
$$


proper $\mathbb{Z}_{N}$ quantization. The fact that we denoted the holonomy of $\int A^{(1)}$ field as $\ell$ is not an accident, and is tied with the insertion of $\mathbb{Z}_{N}$ generators $\mathrm{U}^{\ell}$ into the partition function (3.9). The partition function of $\mathbb{Z}_{N}$ topological gauge theory can be written as

$$
Z_{\mathrm{top}, p}=\int \mathcal{D} A^{(1)} \mathcal{D} A^{(0)} \mathcal{D} F^{(0)} e^{\mathrm{i} \int F^{(0)} \wedge\left(N A^{(1)}-\mathrm{d} A^{(0)}\right)+\mathrm{i} p \int A^{(1)}}
$$

where $F^{(0)}$ is Lagrange multiplier, which forces (3.14), and ip $\int A^{(1)}$ is the Chern-Simons term in $1 d$. The action has gauge redundancy $A^{(1)} \mapsto A^{(1)}+\mathrm{d} \lambda^{(0)}, A^{(0)} \mapsto A^{(0)}+N \lambda^{(0)}$ and $F^{(0)} \mapsto F^{(0)}$.

To couple dynamical field $x$ to the $\mathbb{Z}_{N}$ background field, we declare

$$
x \mapsto x-\lambda^{(0)} .
$$

As a result, the gauge invariant combinations are $N x+A^{(0)}, \mathrm{d} x+A^{(1)}$, and only they can appear in the Lagrangian with a classical $\mathbb{Z}_{N}$ background. Indeed, the insertion of translation generator (3.9) needs to be identified with $Z\left[\left(A^{(1)}, A^{(0)}\right)\right]$ where background $\mathbb{Z}_{N}$ field is given in (3.14).

$$
\begin{aligned}
Z\left[\left(A^{(1)}, A^{(0)}\right)\right] & =\int_{\mathrm{pbc}} \mathcal{D} x e^{-\frac{1}{g} \int d \tau\left(\frac{1}{2}\left(\dot{x}+A_{\tau}\right)^{2}-\cos \left(N x+A^{(0)}\right)\right)+\frac{\mathrm{i} \theta}{2 \pi} \int\left(\mathrm{d} x+A^{(1)}\right)} \delta\left(N A^{(1)}-\mathrm{d} A^{(0)}\right) \\
& =\int_{\tilde{x}(\beta)=\tilde{x}(0)+\frac{2 \pi}{N} \ell} \mathcal{D} \tilde{x} e^{-\frac{1}{g} \int d \tau\left(\frac{1}{2}(\dot{\tilde{x}})^{2}-\cos (N \tilde{x})\right)+\frac{\mathrm{i} \theta}{2 \pi} \int \mathrm{d} \tilde{x}} \\
& =\operatorname{tr}\left[e^{-\beta H} \mathrm{U}^{\ell}\right]=Z_{\ell}(\beta)
\end{aligned}
$$

where pbc denotes periodic boundary conditions $x(\beta)=x(0)$. In the second line, we converted the background $\mathbb{Z}_{N}$ gauge field into a twisted boundary condition for the path integral, by a field redefinition. In the semi-classical description, this guarantees that the leading saddle configuration that contributes to this sum is a fractional instanton with topological charge $\ell / N$.

As described above, gauging $\mathbb{Z}_{N}$ symmetry amounts to summing over all topological gauge theory backgrounds. Moreover, we are allowed to add a topological phase to each network configuration of the topological defects, which is 1d Chern-Simons term. As a result,

$$
\begin{aligned}
\widetilde{Z}_{p}(\beta) \equiv Z_{\left(T_{N} / \mathbb{Z}_{N}\right)_{p}} & =\int \mathcal{D} A^{(1)} \mathcal{D} A^{(0)} \delta\left(N A^{(1)}-\mathrm{d} A^{(0)}\right) Z\left[\left(A^{(1)}, A^{(0)}\right)\right] e^{\mathrm{i} p \int A^{(1)}} \\
& =\frac{1}{N} \sum_{\ell=0}^{N-1} e^{-\mathrm{i} \frac{2 \pi \ell p}{N}} Z_{\ell}
\end{aligned}
$$

This corresponds to the path integral formulation for the theory with the discrete theta angle $\theta_{p}$ or equivalently, the Hilbert space projected to $\mathcal{H}_{p}, p=0,1, \ldots, N-1$.

The $p$ label that permeates the discussion has multiple equivalent and useful interpretations $[69,83,84] .1$ ) Discrete theta angle $\left.\theta_{p}, 2\right)$ Level of Chern-Simons term 3) Decomposition of Hilbert space using projection operators $\Pi_{p}$, where $p$ is Bloch momenta. The 
factorized terms in the exact quantization condition in the $T_{N}$ model can be interpreted as the exact quantization for the $\left(T_{N} / \mathbb{Z}_{N}\right)_{p}$ models.

It is important to note that the local dynamics and saddles in the original $T_{N}$ model whose target space is $S^{1}$ and gauged $\left(T_{N} / \mathbb{Z}_{N}\right)_{p}$ models whose target space is $S^{1} / \mathbb{Z}_{N}$ are same. An instanton with winding number 1 and action $S_{0}$ in the $\left(T_{N} / \mathbb{Z}_{N}\right)$ model is what we would call a fractional instanton with winding number $\frac{1}{N}$ and the same action $S_{0}$ in the original $T_{N}$ model. In this sense, it is natural that exact quantization conditions and perturbative/non-perturbative relations are related in a precise sense. One utility of this perspective is that the mixed anomalies are naturally encoded into exact WKB analysis. Another utility is the perspective that resurgence is valid within the semi-classical description of each $\widetilde{Z}_{p}(\beta) \equiv Z_{\left(T_{N} / \mathbb{Z}_{N}\right)_{p}}$, and this implies that resurgence is closed within each fixed topological sector $Z_{\ell}$ as discussed in section 4.3.

\section{Analysis of the degenerate Weber-type Stokes graphs}

\subsection{Relation between Airy-type and degenerate Weber-type Stokes graphs}

In this section, we investigate the quantization condition for the periodic potential (the $S^{1}$ system) by use of the degenerate Weber(DW)-type Stokes graph. The DW-type Stokes graph should be used under the assumption that the energy spectrum is classically zero $\left(E_{0}=0\right)$. Here, $E_{0}$ is a control parameter of the Stokes graph, and the picture of the Airytype Stokes graph is not naively applicable when taking $E_{0}=0$ where two turning points merge into one. Due to the dependence of the Stokes graph on the value of $E_{0}$, two turning points giving a primary perturbative cycle in the Airy-type Stokes graph collides with each other and merge into a single turning point. The perturbative cycles are given by a residue integration around the merged turning points consequently, while nonperturbative cycles is defined in the same manner as that of the Airy-type graph. For the DW-type Stokes graph, one has to employ the connection formula obtained by the DW-type Schrödinger equation given by

$$
\left[-\hbar^{2} \frac{\partial^{2}}{\partial y^{2}}+\frac{y^{2}}{4}-\hbar \kappa\right] \widehat{\psi}(y, \hbar)=0,
$$

with $\kappa$ being determined by the global potential.

Let us address the relation between the quantization conditions based on the Airy-type and the DW-type Stokes graphs. By rescaling the energy as $E \rightarrow \hbar E$ in the Schrödinger equation, the Stokes graph of the system transforms from the Airy-type to the DW-type as discussed in $[8,45]$, and the monodromy matrix in the DW-type leads to a different connection formula from the Airy-type. The derivation of the connection formula of the DW-type is given in appendix A.1, which is obtained by starting with the DW equation and performing the coordinate transformation.

The connection formula in the Airy- and DW-type seem to be different from each other, but the most important fact we show in this section is that there is a dictionary to translate expressions of cycles from one into the other, which we derive in appendix A.2. Owing to the dictionary, one can immediately find, not only that the DDP formula and 


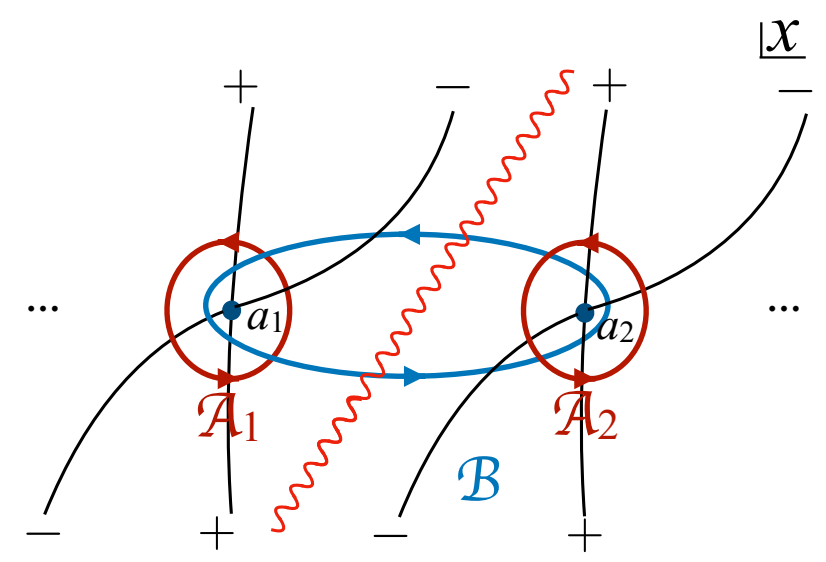

Figure 6. The local degenerate Weber-type Stokes graph is depicted. We here consider two different $\mathcal{A}$ cycles for generality, but they are identical for our present system with periodic potential.

the resurgent structure are unchanged, but also that the techniques such as the resolvent method and the Gutzwiller formula can be performed just by the substitution:

$$
\text { Airy }:(A, B) \longrightarrow \text { Degenerate Weber }:(\mathcal{A}, \mathcal{B})
$$

and following the dictionary shown in table. 1.

Although the two expressions of $D(E)$ derived from Airy-type and DW-type Stokes graphs give equivalent Fredholm determinants, they have their own advantages and disadvantages due to simple fact that different information are encoded in different places. The former gives a clear Gutzwiller representation and makes it easier to see the relationship between the respective quantization methods, but to solve $D(E)=0$ for $E$ accurately is difficult. The latter gives an accurate expression of quasi-moduli-integral and bion amplitude because the construction zoom into $\left(E_{0}=0\right)$ accurately, but the connection formula becomes relatively complicated.

In the next subsection, we discuss the quantization condition by the exact-WKB analysis with the degenerate-Weber-type(DW-type) Stokes graph.

\subsection{From quantization condition to partition function}

Let us begin with showing the connection formula of the DW Stokes graph. We now consider the local Stokes graph as shown in figure 6, where the cycles are denoted as $\mathcal{A}$ and $\mathcal{B}$. For generality, we denote two different $\mathcal{A}$ as $\mathcal{A}_{1}$ and $\mathcal{A}_{2}$, but they are identical in our present problem with the periodic potential.

From now, we consider the potential $V(x)=1-\cos (N x)$, which has $\mathbb{Z}_{N}$ symmetry. In the DW type Stokes graph, the region around a turning point is separated to four regions, that we denote as I, II, III and IV, as shown in figure 7. This is unlike Airy type graphs 


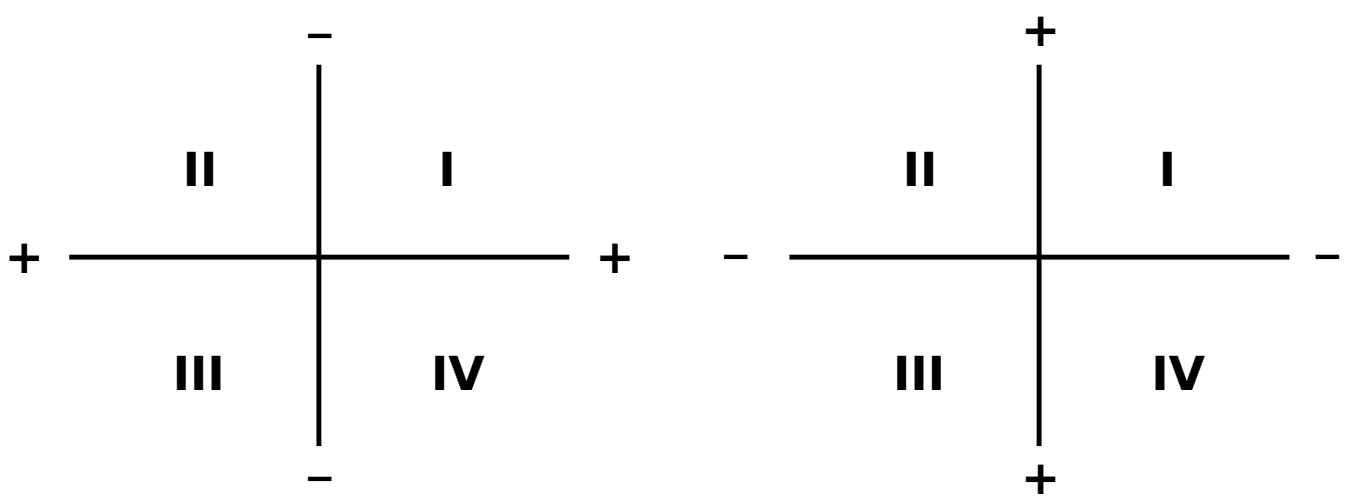

Figure 7. The Stokes curve for the degenerate Weber equation. \pm labels the asymptotics of $\widehat{S}_{\text {odd },-1}(y)$.

which splits to three regions. The monodromy matrices are given by $[44,48]$

$$
\begin{aligned}
\mathcal{M}_{\ominus}^{\mathrm{IV} \rightarrow \mathrm{I}} & =\left(\begin{array}{cc}
1 & 0 \\
i \frac{C_{-}(E, \hbar)}{C_{+}(E, \hbar)} \frac{\sqrt{2 \pi} e^{+\pi i F(E, \hbar)} \hbar^{+F(E, \hbar)}}{\Gamma(1 / 2-F(E, \hbar))} & 1
\end{array}\right), \\
\mathcal{M}_{\oplus}^{\mathrm{I} \rightarrow \mathrm{II}} & =\left(\begin{array}{ll}
1 i \frac{C_{+}(E, \hbar)}{C_{-}(E, \hbar)} \frac{\sqrt{2 \pi} \hbar^{-F(E, \hbar)}}{\Gamma(1 / 2+F(E, \hbar))} \\
0
\end{array}\right), \\
\mathcal{M}_{\ominus}^{\mathrm{II} \rightarrow \mathrm{III}} & =\left(\begin{array}{cc}
1 & 0 \\
i \frac{C_{-}(E, \hbar)}{C_{+}(E, \hbar)} \frac{\sqrt{2 \pi} e^{-\pi i F(E, \hbar)} \hbar^{+F(E, \hbar)}}{\Gamma(1 / 2-F(E, \hbar))} & 1
\end{array}\right), \\
\mathcal{M}_{\oplus}^{\mathrm{III} \rightarrow \mathrm{IV}} & =\left(\begin{array}{ll}
1 i \frac{C_{+}(E, \hbar)}{C_{-}(E, \hbar)} \frac{\sqrt{2 \pi} e^{-2 \pi i F(E, \hbar)} \hbar^{-F(E, \hbar)}}{\Gamma(1 / 2+F(E, \hbar))} \\
0
\end{array}\right),
\end{aligned}
$$

The derivation of monodromy matrices is given in appendix A. Here, $F(E, \hbar)$ are $C_{ \pm}(E, \hbar)$ are defined as

$$
\begin{aligned}
F(E, \hbar) & :=\operatorname{Res}_{x=0} S_{\text {odd }}^{\mathrm{DW}}(x, E, \hbar) \approx-\frac{E}{N} \\
C_{ \pm}(E, \hbar) & :=\lim _{x \rightarrow 0}\left(\frac{\partial y(x, E, \hbar)}{\partial x}\right)^{1 / 2} \frac{\psi_{ \pm}(x, E, \hbar)}{\widehat{\psi}_{ \pm}(y(x, E, \hbar), E, \hbar)} \approx\left(\frac{32}{N}\right)^{\mp \frac{E}{2 N}}
\end{aligned}
$$

respectively, where $a_{\ell}$ is a turning point connecting with its Stokes line. $C_{\ell \pm}(E, \hbar)$ comes from the local coordinate transformation from the DW-type Schrödinger equation in eq. (4.1). $F(E, \hbar)$ is directly related with the "quantum" frequency as $\omega_{\mathcal{A}}(E, \hbar)=$ $-E / F(E, \hbar)$ and $\omega_{\mathcal{A}}(E, \hbar)=N+O(\hbar)$ in the present system. We also define the normalization matrix (Voros multiplier, which accounts the change of turning points in the WKB wave-function) and the branch-cut matrix as

$$
\begin{aligned}
\mathcal{N}_{a_{1}, a_{2}} & :=\left(\begin{array}{cc}
e^{+\frac{1}{\hbar} \int_{a_{1}}^{a_{2}} d x S_{\text {odd },-1}^{\mathrm{DW}}(x)} & 0 \\
0 & e^{-\frac{1}{\hbar} \int_{a_{1}}^{a_{2}} d x S_{\text {odd },-1}^{\mathrm{DW}}(x)}
\end{array}\right), \\
\mathcal{T} & :=\left(\begin{array}{cc}
0 & -i \\
-i & 0
\end{array}\right)
\end{aligned}
$$

respectively, where $a_{1}$ and $a_{2}$ are turning points as $a_{2}=a_{1}+2 \pi \sim a_{1}$. 


\begin{tabular}{|c|c|}
\hline Airy-type & degenerate Weber-type \\
\hline$A_{\ell}=e^{\oint_{A_{\ell}} d x S_{\text {odd }}}$ & $\mathcal{A}_{\ell}=e^{-\frac{2 \pi i E}{\omega_{\mathcal{A}}(E, \hbar)}} \approx e^{-\frac{2 \pi i E}{\omega_{\mathcal{A}_{\ell}}}}$ \\
\hline$B=e^{\oint_{B} d x S_{\text {odd }}}$ & $\mathcal{B}=2 \pi e^{-\frac{S_{B}}{\hbar}} \prod_{\ell=1}^{2} \frac{C_{\ell-}(E, \hbar)}{C_{\ell+}(E, \hbar)} \frac{e^{(-1)^{\ell} \frac{\pi i E}{\omega_{\mathcal{A}_{\ell}}(E, \hbar)}} \hbar^{-\frac{E}{\omega_{\mathcal{A}_{\ell}}(E, \hbar)}}}{\Gamma\left(\frac{1}{2}+\frac{E}{\omega_{\mathcal{A}_{\ell}}(E, \hbar)}\right)}$ \\
& $\approx 2 \pi e^{-\frac{S_{B}}{\hbar}} \prod_{\ell=1}^{2} \frac{e^{(-1)^{\ell} \frac{\pi i E}{\omega_{\mathcal{A}}}}}{\Gamma\left(\frac{1}{2}+\frac{E}{\omega_{\mathcal{A}_{\ell}}}\right)}\left(\frac{N \hbar}{32}\right)^{-\frac{E}{\omega_{\mathcal{A}}}}$ \\
\hline
\end{tabular}

Table 1. Dictionary of $A$ and $B$ cycles between the Airy-type and the DW type. The quantum frequency $\omega_{\mathcal{A}}(E, \hbar)$ has the relationship with $F(E, \hbar)$ in eq. (4.4) as $\omega_{\mathcal{A}}(E, \hbar)=-E / F(E, \hbar) \approx$ $\omega_{\mathcal{A}}+O(\hbar)$. Furthermore, $S_{B}=2 \int_{a_{1}}^{a_{2}} \sqrt{2 V(x)} d x \in \mathbb{R}_{>0}$ is the classical bion action. For the periodic potential, all of $A$-cycles are identical.

The quantization condition for the $T_{N}$ model with $\mathbb{Z}_{N}$ global symmetry is expressed as

$$
\mathcal{D}^{(N) \pm}=\operatorname{det}\left[\left(\mathcal{M}^{ \pm}\right)^{N}-I e^{i \theta}\right]=0
$$

where $\theta$ is the contribution from the $\theta$-angle (or the twisted boundary condition applied to WKB wave-function), and

$$
\mathcal{M}^{+}=\mathcal{M}_{\oplus}^{\mathrm{III} \rightarrow \mathrm{IV}} \mathcal{N}_{a_{1}, a_{2}} \mathcal{T} \mathcal{M}_{\ominus}^{\mathrm{II} \rightarrow \mathrm{III}}, \quad \mathcal{M}^{-}=\mathcal{M}_{\oplus}^{\mathrm{III} \rightarrow \mathrm{IV}} \mathcal{M}_{\ominus}^{\mathrm{IV} \rightarrow \mathrm{I}} \mathcal{N}_{a_{1}, a_{2}} \mathcal{T}
$$

Thus, $\mathcal{D}^{(N) \pm}$ can be expressed as

$$
\mathcal{D}^{(N) \pm}=\frac{1}{\left(\mathcal{A}^{\mp 1} \mathcal{B}\right)^{N / 2}} \prod_{p=0}^{N-1}\left[1+\mathcal{A}^{\mp 1}(1+\mathcal{B})-2 \sqrt{\mathcal{A}^{\mp 1} \mathcal{B}} \cos \left(\frac{\theta+2 \pi p}{N}\right)\right] .
$$

Here, the symbolic notation of cycles, $\mathcal{A}$ and $\mathcal{B}$, are expressed as

$$
\begin{aligned}
& \mathcal{A}:=e^{2 \pi i F} \approx e^{-2 \pi i E / N} \\
& \mathcal{B}:=\left(\frac{C_{-}}{C_{+}}\right)^{2} \frac{2 \pi \mathcal{B}_{0} \hbar^{2 F}}{\Gamma\left(\frac{1}{2}-F\right)^{2}} \approx \frac{2 \pi \mathcal{B}_{0}}{\Gamma\left(\frac{1}{2}+\frac{E}{N}\right)^{2}}\left(\frac{N \hbar}{32}\right)^{-\frac{2 E}{N}}
\end{aligned}
$$

where $\mathcal{B}_{0}=e^{-\frac{16}{N \hbar}}$ denotes the exponential of (minus) action of the bion.

Now, we discuss the relation between the Airy- and the DW-type quantization conditions. Comparing Airy-type and DW type quantization conditions, one finds the dictionary in table 1, where $D^{ \pm}(E)$ obtained from Airy-type and $\mathcal{D}^{ \pm}(E)$ obtained from DW-type are symbolically identical. In other words, if we apply the dictionary in table 1 to $D(E)^{ \pm}$ obtained from Airy-type, we easily obtain the Weber-type one. The derivation of the dictionary is reviewed in appendix A.2. This dictionary is applicable to the generic onedimensional potentials, not only periodic ones.

We here investigate the DW type quantization condition in eq. (4.10) in detail. We first derive the nonperturbative contribution to the ground state energy. Based on the 
approximations in eqs. (4.11) and (4.12), the quantization condition for $N=1,2$ are expressed as

$$
\begin{aligned}
\mathcal{D}^{(1) \pm}(E) \approx & \frac{1}{\sqrt{\mathcal{B}_{0}} \Gamma\left(\frac{1}{2}-E\right)}\left(\frac{\hbar}{32}\right)^{E}+\frac{\sqrt{\mathcal{B}_{0}} e^{ \pm \pi i E}}{\Gamma\left(\frac{1}{2}+E\right)}\left(\frac{\hbar}{32}\right)^{-E}-\sqrt{\frac{2}{\pi}} \cos \theta=0, \\
\mathcal{D}^{(2) \pm}(E) \approx & {\left[\frac{1}{\sqrt{\mathcal{B}_{0}} \Gamma\left(\frac{1}{2}-\frac{E}{2}\right)}\left(\frac{\hbar}{16}\right)^{\frac{E}{2}}+\frac{\sqrt{\mathcal{B}_{0}} e^{ \pm \pi i \frac{E}{2}}}{\Gamma\left(\frac{1}{2}+\frac{E}{2}\right)}\left(\frac{\hbar}{16}\right)^{-\frac{E}{2}}+\sqrt{\frac{2}{\pi}} \cos \frac{\theta}{2}\right] } \\
& \cdot\left[\frac{1}{\sqrt{\mathcal{B}_{0}} \Gamma\left(\frac{1}{2}-\frac{E}{2}\right)}\left(\frac{\hbar}{16}\right)^{\frac{E}{2}}+\frac{\sqrt{\mathcal{B}_{0}} e^{ \pm \pi i \frac{E}{2}}}{\Gamma\left(\frac{1}{2}+\frac{E}{2}\right)}\left(\frac{\hbar}{16}\right)^{-\frac{E}{2}}-\sqrt{\frac{2}{\pi}} \cos \frac{\theta}{2}\right]=0,
\end{aligned}
$$

up to an irrelevant overall factor. Now, we would set the energy as $E / N=1 / 2+\delta$ with $0<|\delta| \ll 1$. Solving the quantization condition in terms of $\delta$ yields

$$
\begin{aligned}
& \delta^{N=1}=-\sqrt{\frac{64 \mathcal{B}_{0}}{\pi \hbar}} \cos \theta+\frac{64 \mathcal{B}_{0}}{\pi \hbar}\left[\cos ^{2} \theta \cdot\left(\gamma-\log \frac{\hbar}{32}\right) \pm \frac{\pi i}{2}\right]+O\left(\mathcal{B}_{0}^{3 / 2}\right) \\
& \delta^{N=2}=-(-1)^{p} \sqrt{\frac{32 \mathcal{B}_{0}}{\pi \hbar}} \cos \frac{\theta}{2}+\frac{32 \mathcal{B}_{0}}{\pi \hbar}\left[\cos ^{2} \frac{\theta}{2} \cdot\left(\gamma-\log \frac{\hbar}{16}\right) \pm \frac{\pi i}{2}\right]+O\left(\mathcal{B}_{0}^{3 / 2}\right),
\end{aligned}
$$

where $\gamma$ is the Euler constant, and $p \in\{0,+1\}$ is the eigenvalue of the $\mathbb{Z}_{2}$-shift symmetry generator. For $N=1$, the first term is the contribution of instanton $[I]$ and anti-instanton $[\bar{I}]$. The second term can be viewed as the contributions of correlated events, $[I I],[\bar{I} \bar{I}]$, and $[I \bar{I}]_{ \pm}$. The imaginary ambiguity originates in $O\left(\mathcal{B}_{0}\right)$ and is sourced by the $[I \bar{I}]_{ \pm}$critical point at infinity. This contribution is cancelled with the same ambiguity that arise from the lateral Borel resummation of perturbation theory. the quantization condition for $N=1$ in the low energy limit in eq. (4.13) is in exact agreement with the result by Zinn-Justin and Jentsuchura [8] up to rescaling parameters in the theory.

For $N=2$, there are two types of fractional instantons, $\left[I_{1}\right],\left[I_{2}\right]$ and $\left[\bar{I}_{1}\right],\left[\bar{I}_{2}\right]$, and at second order, there are $\left[I_{1} I_{2}\right],\left[\bar{I}_{1} \bar{I}_{2}\right],\left[I_{1} \bar{I}_{1}\right]_{ \pm},\left[I_{2} \bar{I}_{2}\right]_{ \pm}$critical points at infinity, whose manifestation in (4.16) is clear. In addition to these similar effects with the $N=1$ case, there is one more interesting phenomenon. At $\theta=\pi$, degenerate energies independent of $p$ is obtained. In fact, the whole Hilbert space is two-fold degenerate. As discussed in the previous section, this is a manifestation of mixed anomaly between the two global symmetries of the theory, $\mathbb{Z}_{2} \times \mathbb{Z}_{2}$, translation and charge conjugation, and anomaly implies the spontaneous breaking down to $\mathbb{Z}_{2}$ leading to two-vacua.

Let us now express the partition function by use of the resolvent method. We restrict to $N=1$ case for simplicity. We separate the partition function into the perturbative and nonperturbative parts as

$$
Z(\hbar, \beta)=Z_{\mathrm{pt}}(\hbar, \beta)+Z_{\mathrm{np}}(\hbar, \beta) .
$$

Since one finds that

$$
\begin{aligned}
\mathcal{D}^{(1) \pm} & \propto 1+\mathcal{A}^{\mp 1}[1+\mathcal{B}]-2 \sqrt{\mathcal{A}^{\mp 1} \mathcal{B}} \cos \theta \\
& =\mathcal{D}_{\mathcal{A}}^{\mp}\left[1+\frac{\mathcal{A}^{\mp 1}}{\mathcal{D}_{\mathcal{A}}^{\mp}} \mathcal{B}-\frac{2 \sqrt{\mathcal{A}}^{\mp 1}}{\mathcal{D}_{\mathcal{A}}^{\mp}} \sqrt{\mathcal{B}} \cos \theta\right],
\end{aligned}
$$


where

$$
\mathcal{D}_{\mathcal{A}}^{ \pm}(E, \hbar)=1+\mathcal{A}(E, \hbar)^{ \pm 1}=1+e^{\mp 2 \pi i \frac{E}{\omega_{\mathcal{A}}(E, \hbar)}}=\frac{2 \pi e^{\mp \pi i \frac{E}{\omega_{\mathcal{A}}(E, \hbar)}}}{\Gamma\left(\frac{1}{2}+\frac{E}{\omega_{\mathcal{A}}(E, \hbar)}\right) \Gamma\left(\frac{1}{2}-\frac{E}{\omega_{\mathcal{A}}(E, \hbar)}\right)} .
$$

The perturbative part can be found easily as

$$
Z_{\mathrm{pt}}^{N=1}(\hbar, \beta)=\frac{1}{2 \pi i} \int_{\epsilon-i \infty}^{\epsilon+i \infty}\left[-\frac{\partial \log \mathcal{D}_{\mathcal{A}}^{\mp}}{\partial E}\right] e^{-\beta E} d E,
$$

and the nonperturbative part can be written as the expanded form in terms of the instanton and bion contributions as:

$$
\begin{aligned}
& Z_{\mathrm{np}}^{N=1}(\hbar, \beta)=\frac{1}{2 \pi i} \int_{\epsilon-i \infty}^{\epsilon+i \infty}\left[-\frac{\partial}{\partial E} \log \left(1+\frac{\mathcal{A}^{\mp 1}}{\mathcal{D}_{\mathcal{A}}^{\mp}} \mathcal{B}-\frac{2 \sqrt{\mathcal{A}}^{\mp 1}}{\mathcal{D}_{\mathcal{A}}^{\mp}} \sqrt{\mathcal{B}} \cos \theta\right)\right] e^{-\beta E} d E \\
&= \frac{\beta}{2 \pi i} \int_{\epsilon-i \infty}^{\epsilon+i \infty} \sum_{n=1}^{\infty} \sum_{m=0}^{n} \frac{1}{n}\left(\begin{array}{c}
n \\
m
\end{array}\right)\left(-\frac{\mathcal{A}^{\mp 1}}{\mathcal{D}_{\mathcal{A}}^{\mp}} \mathcal{B}\right)^{m}\left(\frac{\sqrt{\mathcal{A}}^{\mp 1}}{\mathcal{D}_{\mathcal{A}}^{\mp}} \sqrt{\mathcal{B}}\left(e^{i \theta}+e^{-i \theta}\right)\right)^{n-m} e^{-\beta E} d E \\
&= \sum_{\substack{(Q, K) \in \mathbb{Z} \otimes \mathbb{N}_{0} \\
|Q|+K>0}} Z_{\mathrm{np}}^{N=1}(\hbar, \beta ;\{Q, K\}), \\
& Z_{\mathrm{np}}^{N=1}(\hbar, \beta ;\{Q, K\}):=\frac{\beta}{2 \pi i} \int_{\epsilon-i \infty}^{\epsilon+i \infty} \frac{1}{|Q|+K}\left(\begin{array}{c}
|Q|+K \\
K
\end{array}\right)\left(\frac{\mathcal{B}}{\mathcal{K}^{2}}\right)^{|Q| / 2+K} \\
& \cdot{ }_{2} F_{1}\left(1-K,-K ;|Q|+1 ;-\mathcal{A}^{ \pm 1}\right)\left(-\mathcal{A}^{\mp 1}\right)^{K} e^{-\beta E+i Q \theta} d E,
\end{aligned}
$$

where $Q$ and $K$ are the topological charge and the number of bions, respectively, and

$$
\mathcal{K}:=\sqrt{\mathcal{A}}^{+1}+\sqrt{\mathcal{A}}^{-1}=\mathcal{D}_{\mathcal{A}}^{ \pm} \sqrt{\mathcal{A}}^{\mp 1} .
$$

Using table 1, we can get the quasi-moduli-integral (QMI) form:

$$
\begin{aligned}
Z_{\mathrm{np}}^{N=1}(\hbar, \beta ;\{Q, K\})= & \frac{\beta}{2 \pi i} \int_{\epsilon-i \infty}^{\epsilon+i \infty} \frac{(-1)^{K}}{|Q|+K}\left(\begin{array}{c}
|Q|+K \\
K
\end{array}\right)\left[\frac{e^{-\frac{S_{B}}{\hbar}}}{2 \pi} \Gamma\left(\frac{1}{2}-\frac{E}{\omega_{\mathcal{A}}}\right)^{2}\left(\frac{\hbar}{32}\right)^{-\frac{2 E}{\omega_{\mathcal{A}}}}\right]^{|Q| / 2+K} \\
& \cdot{ }_{2} F_{1}\left(1-K,-K ;|Q|+1 ;-e^{\mp 2 \pi i \frac{E}{\omega_{\mathcal{A}}}}\right)\left(e^{ \pm 2 \pi i \frac{E}{\omega_{\mathcal{A}}}}\right)^{K} e^{-\beta E+i Q \theta} d E .
\end{aligned}
$$

The physical meaning of each term is as follows: $\beta$ is the exact zero mode of the bion and instanton, $(-1)^{K}$ is the Maslov index, $\left(\begin{array}{c}|Q|+K \\ K\end{array}\right)$ and $\frac{1}{|Q|+K}$ are combination and cyclic permutation of $K$-bions and $Q$-instantons, $\left(\frac{e^{-\frac{S_{B}}{\hbar}}}{2 \pi} \Gamma\left(\frac{1}{2}-\frac{E}{\omega}\right)^{2}\left(\frac{\hbar}{32}\right)^{-\frac{2 E}{\omega}}\right)^{|Q| / 2+K}$ is nothing but the bion (and instanton) amplitude and QMI integral. Notice that the label of phase ambiguity is $K$, which is the number of (neutral) bions, not the instanton.

For generic $N$, the partition function can be written in the way parallel to the case of $N=1$. Since both of perturbative and nonperturbative contributions is $N$-times of that 
for the single-periodic case, the nonperturbative part can be written as

$$
\begin{aligned}
Z_{\mathrm{np}}(\hbar, \beta)= & \sum_{p=0}^{N-1} \sum_{\substack{\left(Q_{p}, K_{p}\right) \in \mathbb{Z} \otimes \mathbb{N}_{0} \\
\left|Q_{p}\right|+K_{p}>0}} Z_{\mathrm{np}}\left(\hbar, \beta ;\left\{p, Q_{p}, K_{p}\right\}\right) \\
= & N \sum_{\substack{(Q, K) \in \mathbb{Z} \otimes \mathbb{N}_{0} \\
|Q|+K>0}} Z_{\mathrm{np}}(\hbar, \beta ;\{0, N Q, K\}), \\
Z_{\mathrm{np}}\left(\hbar, \beta ;\left\{p, Q_{p}, K_{p}\right\}\right):= & \frac{\beta}{2 \pi i} \int_{\epsilon-i \infty}^{\epsilon+i \infty} \frac{e^{2 \pi i p Q_{p} / N}}{\left|Q_{p}\right|+K_{p}}\left(\begin{array}{c}
\left|Q_{p}\right|+K_{p} \\
K_{p}
\end{array}\right)\left(\frac{\mathcal{B}}{\mathcal{K}^{2}}\right)^{\left|Q_{p}\right| / 2+K_{p}} \\
& \cdot{ }_{2} F_{1}\left(1-K_{p},-K_{p} ;\left|Q_{p}\right|+1 ;-\mathcal{A}^{ \pm 1}\right)\left(-\mathcal{A}^{\mp 1}\right)^{K_{p}} e^{-\beta E+i Q_{p} \theta / N} d E .
\end{aligned}
$$

Here, in order to derive eq. (4.26), we have performed the discrete Fourier transform in eq. (4.25), ${ }^{5}$ and consequently the contribution from $Q_{p} \in N \mathbb{Z}$ remains. This fact is directly seen in the partition function given through the resolvent method. For example, by employing the result in eq. (4.16), the partition function for $N=2$ with the nonperturbative contribution can be approximately estimated by

$$
\begin{aligned}
\left.Z^{N=2}(\hbar, \beta)\right|_{\text {ground state }} & \approx \sum_{p=0}^{1} e^{-2 \beta\left(\frac{1}{2}+\delta_{p}^{N=2}\right)} \\
& =2 e^{-\beta}\left[1+\frac{8 \beta \mathcal{B}_{0}}{\pi \hbar}\left\{(1+\cos \theta)\left(\beta+2 \log \frac{\hbar}{16}-2 \gamma\right) \mp 2 \pi i\right\}\right]+O\left(\mathcal{B}_{0}^{2}\right)
\end{aligned}
$$

One can immediately find that there only exists the terms proportional to $\cos (Q \theta)$ with $Q \in \mathbb{N}_{0}$, which means that the contribution from the $Q_{p} \notin N \mathbb{Z}$ sector is cancelled by other $p$-sectors.

\subsection{Resurgent structure of the Hilbert space and the partition function}

Finally, we comment on the resurgent structure of the quantizaion conditions, the partition function, and fixed topological charge sectors of the partition function (which are the columns of resurgence triangle).

We first consider the quantization condition and in order to see the implication of resurgence in fixed discrete theta angle $p$, we rewrite eq. (4.10) as

$$
\begin{aligned}
\mathcal{D}^{(N) \pm} & =\frac{1}{\left(\mathcal{A}^{\mp 1} \mathcal{B}\right)^{N / 2}} \prod_{p=0}^{N-1} \mathcal{D}_{p}^{(N) \pm} \\
& =\left(\alpha^{ \pm}\right)^{N} \prod_{p=0}^{N-1}\left(1-\beta^{ \pm} e^{+i(\theta+2 \pi p) / N}\right)\left(1-\beta^{ \pm} e^{-i(\theta+2 \pi p) / N}\right)
\end{aligned}
$$

\footnotetext{
${ }^{5}$ The subscript $p$ in $Q_{p}$ and $K_{p}$ is a dummy index.
} 
where

$$
\begin{aligned}
\mathcal{D}_{p}^{(N) \pm} & :=1+\mathcal{A}^{\mp 1}(1+\mathcal{B})-2 \sqrt{\mathcal{A}^{\mp 1} \mathcal{B}} \cos \left(\frac{\theta+2 \pi p}{N}\right), \\
\alpha^{ \pm} & =\xi^{ \pm}+\sqrt{\left(\xi^{ \pm}\right)^{2}-1}, \quad \beta^{ \pm}=\xi^{ \pm}-\sqrt{\left(\xi^{ \pm}\right)^{2}-1}, \quad \xi^{ \pm}=\frac{1+\mathcal{A}^{ \pm 1}+\mathcal{B}}{2 \sqrt{\mathcal{A}^{ \pm 1} \mathcal{B}}} .
\end{aligned}
$$

Since the DDP formula gives

$$
\mathcal{S}_{+}[\sqrt{\mathcal{A}}]=\mathcal{S}_{-} \mathfrak{S}[\sqrt{\mathcal{A}}] \quad \Rightarrow \quad \mathcal{S}_{+}\left[\xi^{+}\right]=\mathcal{S}_{-}\left[\xi^{-}\right] \quad \Rightarrow \quad\left\{\begin{array}{l}
\mathcal{S}_{+}\left[\alpha^{+}\right]=\mathcal{S}_{-}\left[\alpha^{-}\right] \\
\mathcal{S}_{+}\left[\beta^{+}\right]=\mathcal{S}_{-}\left[\beta^{-}\right]
\end{array}\right.
$$

where $\mathfrak{S}$ denotes the Stokes automorphism defined as $\mathfrak{S}[\sqrt{\mathcal{A}}]=(1+\mathcal{B}) \sqrt{\mathcal{A}}$, one can easily see that the DDP transformation is closed in each of $p$-sectors. This is not surprising, as each one of the $p$ sectors corresponds to $N=1$ system with the replacement of $\theta \rightarrow \frac{\theta+2 \pi p}{N}$. The energy spectrum obtained by solving the quantization condition directly corresponds to the Hilbert space $\mathcal{H}_{p}$, and it means that $\mathcal{H}_{p}$ is invariant under the Stokes automorphism $\mathcal{S}$. At the same time, the partition function can be written through the resolvent method by keeping $\left(\alpha^{ \pm}, \beta^{ \pm}\right)$as ${ }^{6}$

$$
\begin{aligned}
Z^{ \pm}(\hbar, \beta) & =\frac{\beta}{2 \pi i} \int_{\epsilon-i \infty}^{\epsilon+i \infty}\left[-N \log \left(\sqrt{\mathcal{A}^{\mp 1} \mathcal{B}} \alpha^{ \pm}\right)+\sum_{p=0}^{N-1} \sum_{Q_{p} \in \mathbb{Z} \backslash\{0\}} \frac{\left(\beta^{ \pm}\right)^{\left|Q_{p}\right|}}{\left|Q_{p}\right|} e^{i(\theta+2 \pi p) Q_{p} / N}\right] e^{-\beta E} d E \\
& =\frac{\beta}{2 \pi i} \int_{\epsilon-i \infty}^{\epsilon+i \infty}\left[-N \log \left(\sqrt{\mathcal{A}^{\mp 1} \mathcal{B}} \alpha^{ \pm}\right)+\sum_{Q \in \mathbb{Z} \backslash\{0\}} \frac{\left(\beta^{ \pm}\right)^{N|Q|}}{|Q|} e^{i Q \theta}\right] e^{-\beta E} d E
\end{aligned}
$$

The first and second terms in eq. (4.33) correspond to the $Q_{p}=0$ and $Q_{p} \neq 0$ sectors, respectively, and we performed the discrete Fourier transform by summing $p$ up to obtain eq. (4.34). Notice that the DDP invariance for the $Q_{p}=0$ sector can be ensured as

$$
\begin{aligned}
\mathcal{S}_{+}\left[Z_{Q_{p}=0}^{+}(\hbar, \beta)\right] & =-\frac{N \beta}{2 \pi i} \int_{\epsilon-i \infty}^{\epsilon+i \infty} \mathcal{S}_{+}\left[\log \left(\sqrt{\mathcal{A}^{-1} \mathcal{B}} \alpha^{+}\right)\right] e^{-\beta E} d E \\
& =-\frac{N \beta}{2 \pi i} \int_{\epsilon-i \infty}^{\epsilon+i \infty} \mathcal{S}_{-}\left[\log \frac{1}{\mathcal{A}(1+\mathcal{B})}+\log \left(\sqrt{\mathcal{A B}} \alpha^{-}\right)\right] e^{-\beta E} d E \\
& =-\frac{N \beta}{2 \pi i} \int_{\epsilon-i \infty}^{\epsilon+i \infty} \mathcal{S}_{-}\left[\log \left(\sqrt{\mathcal{A B}} \alpha^{-}\right)\right] e^{-\beta E} d E=\mathcal{S}_{-}\left[Z_{Q_{p}=0}^{-}(\hbar, \beta)\right]
\end{aligned}
$$

To drive the third line, we have used the fact that $\log \frac{1}{\mathcal{A}(1+\mathcal{B})}$ is a holomorphic function of $E$. As emphasized earlier, $p$ has few equivalent interpretation. One is the label of Bloch momentum that is eigenstate of the $\mathbb{Z}_{N}$-translation symmetry given by $x \rightarrow x+2 p \pi / N$. The important fact is that the $\mathcal{D}_{p}^{(N) \pm}$ gives the $Q_{p}$-summed partition function which is decomposable into the $Q_{p}$-sectors for the Stoke automorphism. It can be seen from the fact

\footnotetext{
${ }^{6} \log \sqrt{\mathcal{A}^{\mp 1} \mathcal{B}}$ in eq. (4.33) is nothing but a convention to make the perturbative part as $\mathcal{D}_{\mathcal{A}}^{\mp}$ in $\mathcal{D}^{(N) \pm}$. It does not affect the resulting partition function because it disappears in the energy integration.
} 
that $\sum_{K_{p}} Z\left(\beta ;\left\{p, Q_{p}, K_{p}\right\}\right)$ by eq. (4.27) is indeed invariant under the DDP transformation and irreducible in the sense that $Z\left(\beta ;\left\{p, Q_{p}, K_{p}\right\}\right)$ is not invariant. Therefore, the transseries structure of the partition function is characterized not only by the topological charge but also the $\mathbb{Z}_{N}$ translation symmetry. This complete resurgent structure of the partition function is exhibited in figure 8. We summarize the relationship of the Hilbert space and the partition function below:

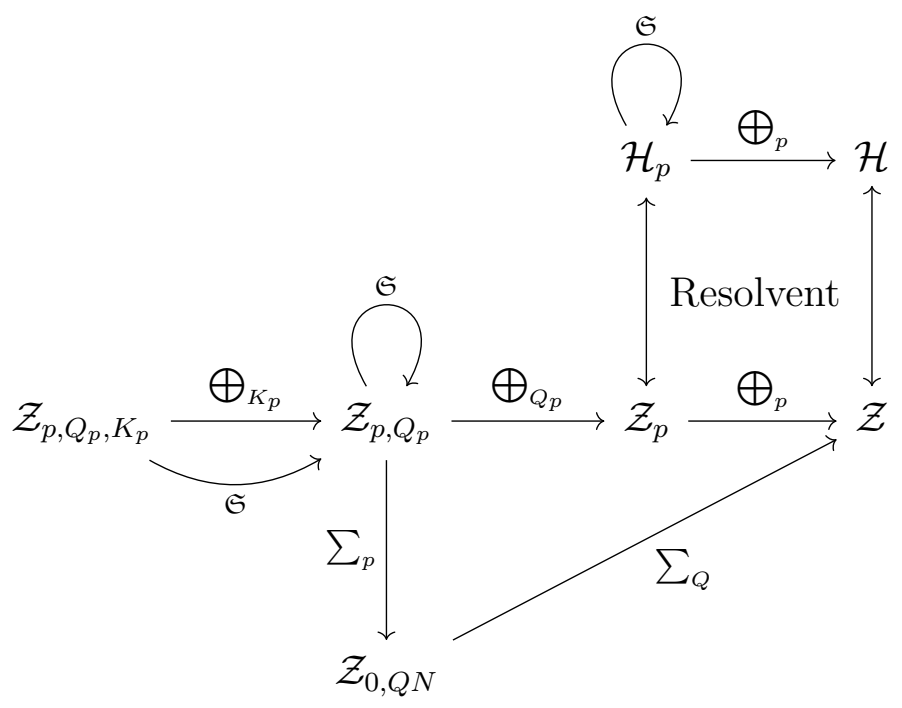

where $Z^{ \pm}\left(\hbar, \beta ;\left\{p, Q_{p}, K_{p}\right\}\right) \in \mathcal{Z}_{p, Q_{p}, K_{p}}, Z^{ \pm}(\hbar, \beta) \in \mathcal{Z}$, and

$$
\begin{aligned}
\mathcal{H}_{p} & :=\left\{|\psi\rangle \in L^{2}(\mathbb{R}): \widehat{H}|\psi\rangle=E_{n, p}|\psi\rangle \text { where } E_{n \in \mathbb{N}_{0}, p} \text { are solutions of } \mathcal{D}_{p}^{N( \pm)}(E)=0\right\}, \\
\mathcal{Z}_{p, Q_{p}, K_{p}} & :=\left\{\mathbb{C}\left[\left[\hbar, \frac{e^{-\frac{S_{B}}{2 \hbar}}}{\hbar^{1 / 2}}, \log \hbar\right]\right]: e^{i\left(Q_{p}+2 \pi p\right) / N} \int_{\epsilon-i \infty}^{\epsilon+i \infty} d E e^{-\beta E} \mathcal{B}^{\left|Q_{p}\right| / 2+K_{p}} \mathbb{C}\left[\left[\mathcal{A}^{\mp 1}\right]\right]\right\} .
\end{aligned}
$$

From eq. (4.36) one can immediately claim that the resurgence in eq. (3.18) is closed within each fixed topological sector of $\tilde{Z}_{p}$ (and $Z_{\ell}$ via the Fourier transform).

Alternative derivation. Consider $T_{1}$ model with theta angle. Exact quantization condition for this system is given in (2.21). The solution of the $D^{ \pm}(E, \theta)=0$ is in correspondence with the Hilbert space $\mathcal{H}_{\theta}$, based on the $\theta$ vacuum,

$$
\left|\Psi_{\theta}\right\rangle=\sum_{n \in \mathbb{Z}} e^{i n \theta}\left|0_{n}\right\rangle
$$

where $\left|0_{n}\right\rangle$ is the harmonic ground state and its copies under large gauge transformation. This perspective follows from the fact that to obtain $T_{1}$ model, we gauged $\mathbb{Z}$ translation symmetry for the particle on a line $x \in \mathbb{R}$, and obtained $x \in S^{1}=\mathbb{R} / 2 \pi \mathbb{Z}$ with $N=1$. (For detailed discussion of particle on a line in periodic potential vs. particle on a circle, see [87])

We showed that the condition $D^{ \pm}(E, \theta)=0$ is invariant under left/right Borel resummation (2.23). This implies that the partition function of the $T_{1}$ system

$$
Z(\theta)=\sum_{Q \in \mathbb{Z}} e^{i \theta Q} \int_{x(\beta)=x(0)+2 \pi Q} D x e^{-S[x]} \equiv \sum_{Q \in \mathbb{Z}} e^{i \theta Q} Z_{Q}
$$




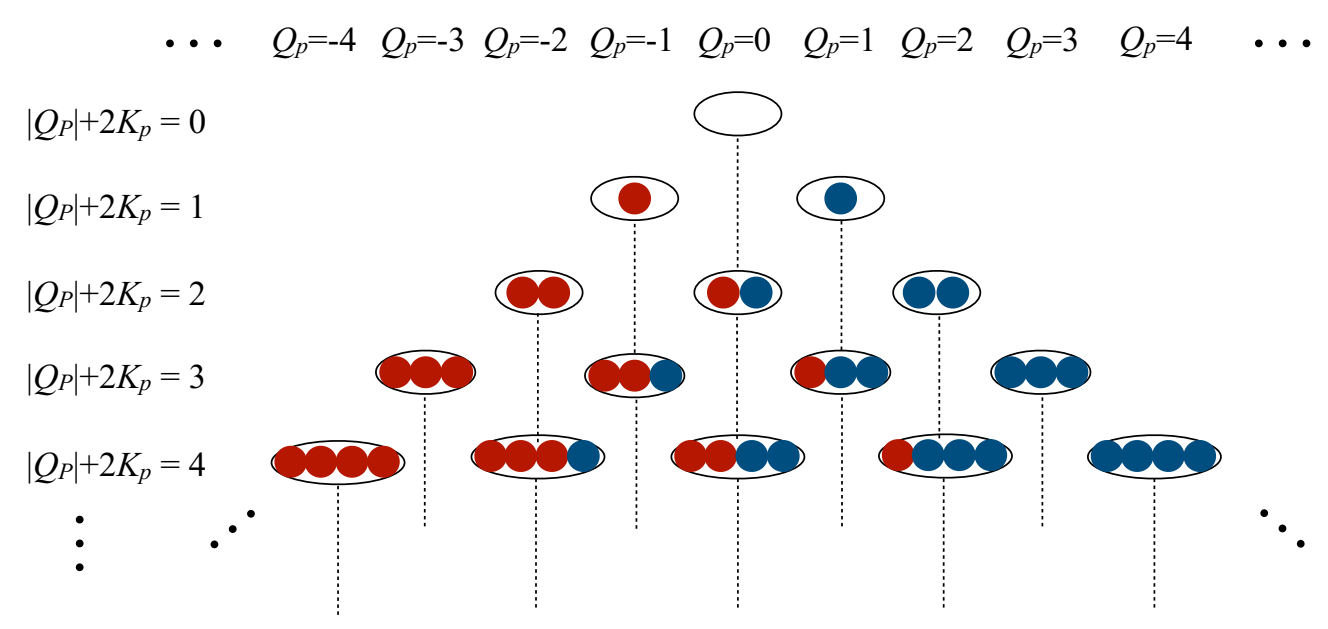

Figure 8. Resurgence triangle exhibiting the complete resurgent structure is depicted, where blue points stand for instantons and red points for anti-instantons. We show the structure for each $p$. Thus, there are $N$ copies of this structure in total. By summing up all of the $p$-sectors, the cancellation among the $p$-sectors arises and the contribution only from the $Q_{p}$-sectors with $Q_{p} \in N \mathbb{Z}$ remains.

is invariant under left/right Borel resummation. The Fourier coefficients correspond to the twisted partition functions,

$$
Z_{Q}=\operatorname{tr}\left[(\mathrm{U})^{Q} e^{-\beta H}\right]=\frac{1}{2 \pi} \int_{0}^{2 \pi} d \theta e^{-i \theta Q} Z(\theta)
$$

which are in one-to-one correspondence with the columns of resurgence triangle. The linearity of the Fourier transform implies that each column of the resurgence triangle, i.e, each fixed topological charge sector, is closed under resurgence. Ambiguities of Borel resummation of perturbation theory around the multi-instanton $\left[I^{k}\right] \sim e^{-k S_{I} / \hbar} P(\hbar)$ are cured by the ambiguity in the amplitude of $\left[I^{k+1} \bar{I}\right]_{ \pm},\left[I^{k+2} \bar{I}^{2}\right]_{ \pm}, \ldots$ events etc.

\section{Summary and discussion}

We have investigated quantum mechanical systems of a particle on $S^{1}$ in the presence of a periodic potential with $N$-minima $(N=1,2 \ldots)$ by the exact-WKB method. We used Stokes graphs with both Airy and degenerate Weber type building blocks, and determined exact quantization conditions. By using the DDP formula that related the perturbative and non-perturbative cycles, we showed invariance of the quantization condition under Stokes automorphism. This implies that all orders perturbative/non-perturbative resurgent cancellations is implicit in the quantization condition and partition function.

The implication of our result for the Gutzwiller trace formula are also discussed. In particular, our construction identifies prime periodic orbits that enter to the trace formula, leading to an understanding of its $\theta$ dependence. Exact-WKB analysis correctly produces the conjectured quantization condition for $N=1$. The symbolic forms of the Fredholm determinant obtained by Airy-type (2.21) and by degenerate Weber-type (4.12) coincide. Our 
result obtained by the degenerate Weber-type correctly reproduces the energy eigenvalues conjectured by Zinn-Justin [7], and obtained earlier by using uniform WKB method in [13] for $N=1$. For general $N$, the resurgent structure is closed in the eigenspaces of $\mathbb{Z}_{N}$-shift symmetry, i.e, $\widetilde{Z}_{p}$ associated with $\mathcal{H}_{p}$ subspace of the Hilbert space. The Fourier transform of this relation gives us the result that resurgent structure is closed in fixed topological charge sectors. This implies that the ambiguity of perturbation theory around an instanton $[I]$ is cured by $[I I \bar{I}]_{ \pm},[I I I \bar{I} \bar{I}]_{ \pm}$etc. which lives on the same topological charge sector. According to DDP formula, this structure is true on all columns of resurgence triangle.

Furthermore, we have also shown that exact quantization condition naturally captures the mixed 't Hooft anomaly [69] or global inconsistency between $\mathbb{Z}_{N}$ translation symmetry and charge conjugation symmetry $C$ [84].

Below, we list few topics to which we may be able to apply our methodology:

- Tilted periodic-potential quantum mechanics, including supersymmetric and quasiexactly solvable cases.

- Constructive resurgence between perturbative/non-perturbative sectors [6, 11] in $S^{1}$ quantum mechanics. This type of resurgence connects different topological sectors to each other, unlike the one we discussed here, which always take place within a fixed topological sector.

- Compactification of QFTs with background fluxes down to quantum mechanics, such as the ones discussed in [70].

- Schwinger mechanism for time-dependent electric field [67], where the Klein-Gordon equation in scalar QED has a Schrödinger-equation form.

Most of readers may be interested in the application of exact-WKB to quantum field theory (not to the reduced quantum mechanics). Toward this goal, we first need to investigate the exact-WKB analysis for quantum mechanics with multiple degrees of freedom, e.g. After completing such extension, we will consider taking the limit of infinite degrees of freedom, which could have an implication to quantum field theory.

\section{Acknowledgments}

The authors especially thank O. Morikawa and students of E-lab for fruitful discussion on quantization conditions for $S^{1}$ quantum mechanics. T.M. is supported by the Japan Society for the Promotion of Science (JSPS) Grant-in-Aid for Scientific Research (KAKENHI) Grant Numbers 18H01217 and 19K03817. S.K. is supported by the Polish National Science Centre grant 2018/29/B/ST2/02457. The original questions related to the present work were posed in "RIMS-iTHEMS International Workshop on Resurgence Theory" at RIKEN, Kobe in 2017. The authors are grateful to the organizers and participants of the workshop. M.U. acknowledges support from U.S. Department of Energy, Office of Science, Office of Nuclear Physics under Award Number DE-FG02-03ER41260. The authors thank Yukawa Institute for Theoretical Physics at Kyoto University. Discussions during 


\begin{tabular}{|c|c|c|c|c|}
\hline & Wavefunction & Classical potential & Riccati var. & Energy \\
\hline Global & $\psi(x, \hbar)$ & $Q(x, \hbar)=2(V(x)-E)$ & $S(x, \hbar)$ & $E$ \\
Local & $\widehat{\psi}(y, \hbar)$ & $\widehat{Q}(y, \hbar)=\frac{y^{2}}{4}-\hbar \kappa$ & $\widehat{S}(y, \hbar)$ & $\kappa / 2$ \\
\hline
\end{tabular}

Table 2. Notation table for global and local variables.

the YITP-RIKEN iTHEMS workshop YITP-T-20-03 on "Potential Toolkit to Attack Nonperturbative Aspects of QFT -Resurgence and related topics-" were useful to complete this work.

\section{A The degenerate Weber equation}

In appendix A.1, we review the derivation of the connection formula for the degenerateWeber type Stokes graph. See pages 28-40 of ref. [49] for the proof of this construction for the Airy type building blocks of Stokes graph (corresponding to $\widehat{Q}(y(x, \hbar), \hbar)=y(x, \hbar)$ below). Appendix A.1 is the generalization of that local/global relations by using degenerate Weber type building blocks of Stokes graph. Appendix A.2 is devoted to the construction of dictionary between the Airy-type and the degenerate Weber-type building blocks. In order to avoid confusion, we summarized the notation for global and local variables in table 2, which are used below.

\section{A.1 Derivation of the connection formula}

As we discussed through the paper, given a classical potential, we can immediately obtain the Stokes graph. The building blocks of the Stokes graph are either Airy type or degenerate Weber type building blocks. In this appendix, we review the derivation of connection formula of the degenerate Weber(DW) equation. In the construction of exact quantization conditions, three important matrices play a role. Connection matrices used for passage through Stokes line, normalization matrix (or Voros multiplier) which accounts for the change of turning point, and branchcut matrices which account for the passage through a branchcut. The combination of these and a global boundary condition on WKB wave function is the exact quantization condition.

In order to obtain the formula, one has to take the two steps: firstly obtaining the connection formula for the local coordinate, and then lifting them up to the global coordinate. The following relations which are equivalent to each others are important:

$$
\begin{aligned}
& \text { (1) } Q(x, \hbar)=\left(\frac{\partial y(x, \hbar)}{\partial x}\right)^{2} \widehat{Q}(y(x, \hbar), \hbar)-\frac{\hbar^{2}}{2}\{y(x, \hbar) ; x\}, \\
& \text { (2) } S^{( \pm)}(x, \hbar)=\frac{\partial y(x, \hbar)}{\partial x} \cdot \widehat{S}^{( \pm)}(y(x, \hbar), \hbar)-\frac{1}{2} \frac{\frac{\partial^{2} y(x, \hbar)}{\partial x^{2}}}{\frac{\partial y(x, \hbar)}{\partial x}}, \\
& \text { (3) } S_{\text {odd }}(x, \hbar)=\frac{\partial y(x, \hbar)}{\partial x} \cdot \widehat{S}_{\text {odd }}(y(x, \hbar), \hbar),
\end{aligned}
$$


where

$$
\begin{aligned}
\{y ; x\} & =\frac{\frac{\partial^{3} y(x, \hbar)}{\partial x^{3}}}{\frac{\partial y(x, \hbar)}{\partial x}}-\frac{3}{2}\left(\frac{\frac{\partial^{2} y(x, \hbar)}{\partial x^{2}}}{\frac{\partial y(x, \hbar)}{\partial x}}\right)^{2}, \\
y(x, \hbar) & =\sum_{n=0}^{+\infty} y_{n}(x) \hbar^{n},
\end{aligned}
$$

$x$ and $y$ are the global and local coordinates, respectively, and $S(x, \hbar) / \widehat{S}(y, \hbar)$ is the global/local asymptotic solution of the Riccati equation with a global/local potential $Q(x, \hbar) / \widehat{Q}(y, \hbar)$,

$$
\begin{aligned}
& S(x, \hbar)=\sum_{n=-1}^{\infty} S_{n}(x) \hbar^{n}, \\
& S_{-1}^{( \pm)}(x)= \pm \sqrt{Q_{0}(x)} \\
& 2 S_{-1}^{( \pm)}(x) S_{n}^{( \pm)}(x)+\sum_{k, \ell=0}^{k+\ell=n-1} S_{k}^{( \pm)}(x) S_{\ell}^{( \pm)}(x)+\frac{d S_{n-1}^{( \pm)}(x)}{d x}=Q_{n+1}(x), \quad n \in \mathbb{N}_{0}, \\
& S_{\text {odd }}(x, \hbar)=\frac{1}{2}\left(S^{(+)}(x, \hbar)-S^{(-)}(x, \hbar)\right), \quad S_{\text {even }}(x, \hbar)=\frac{1}{2}\left(S^{(+)}(x, \hbar)+S^{(-)}(x, \hbar)\right) .
\end{aligned}
$$

From the above relationships, the global and local wavefunctions, $\psi(x, \hbar)$ and $\widehat{\psi}(y, \hbar)$, can be connected as

$$
\psi_{ \pm}(x, \hbar)=C_{ \pm}(\hbar)\left(\frac{\partial y(x, \hbar)}{\partial x}\right)^{-1 / 2} \widehat{\psi}_{ \pm}(y(x, \hbar), \hbar)
$$

where

$$
C_{ \pm}(\hbar)=\sum_{n=0}^{+\infty} C_{ \pm, n} \hbar^{n}
$$

Let us start with the degenerate Weber equation given by

$$
\left[-\hbar^{2} \frac{\partial^{2}}{\partial y^{2}}+\widehat{Q}(y, \hbar)\right] \widehat{\psi}(y, \hbar)=0, \quad \widehat{Q}(y, \hbar)=\frac{y^{2}}{4}-\hbar \kappa, \quad \kappa \in \mathbb{R}
$$

where $y \in \mathbb{C}$ is the local coordinate and $\widehat{\psi}$ is a local wavefunction. By solving the Riccati equation recursively, the formal solution is defined as

$$
\widehat{\psi}_{ \pm}(y, \hbar)=\frac{y^{\mp \kappa} e^{ \pm \hbar^{-1} y^{2} / 4}}{\sqrt{\widehat{S}_{\text {odd }}(y, \hbar)}} \exp \left[ \pm \int_{\infty}^{y} d y\left(\widehat{S}_{\text {odd }}(y, \hbar)-\hbar^{-1} \widehat{S}_{\text {odd },-1}(y)-\widehat{S}_{\text {odd }, 0}(y)\right)\right]
$$

where

$$
\widehat{S}_{\text {odd },-1}(y)=\frac{y}{2}, \quad \widehat{S}_{\text {odd }, 0}(y)=-\frac{\kappa}{y},
$$

and other $\widehat{S}_{\text {odd }, n>0}(y)$ can be computed in the similar way. In eq. (A.13), we took a reference point of the normalization at $y=\infty$ for $\widehat{S}_{\text {odd }, n>0}(y)$. For convenience, we redefine the wave function as

$$
\widehat{u}_{ \pm}(y, \hbar):=\hbar^{ \pm \kappa / 2} \widehat{\psi}_{ \pm}(y, \hbar)
$$


The asymptotic solution can be obtained as ${ }^{7}$

$$
\widehat{u}_{ \pm}(y, \hbar)=\sqrt{2 y} e^{ \pm \hbar^{-1} y^{2} / 4} \sum_{n=0}^{+\infty} \frac{\widehat{u}_{n}^{ \pm}}{y^{1 \pm \kappa+2 n}} \hbar^{(1 \pm \kappa) / 2+n} .
$$

Substituting eq. (A.16) into eq. (A.12) gives the recursion relation for the coefficients $\widehat{u}_{n}^{ \pm}$as

$$
2(n+1) \widehat{u}_{n+1}^{ \pm}= \pm\left(\frac{1}{2} \pm \kappa+2 n\right)\left(\frac{3}{2} \pm \kappa+2 n\right) \widehat{u}_{n}^{ \pm} \quad \text { with } \quad \widehat{u}_{0}^{ \pm}=1,
$$

and the coefficients are obtained as

$$
\widehat{u}_{n}^{ \pm}=( \pm 2)^{n-2} \frac{(1 / 2 \pm \kappa)(3 / 2 \pm \kappa)(5 / 4 \pm \kappa / 2)_{n-1}(7 / 4 \pm \kappa / 2)_{n-1}}{\Gamma(n+1)}
$$

where $(x)_{n}=\Gamma(x+n) / \Gamma(x)$ is the Pochhammer symbol. Acting the Borel transform $\mathcal{B}$ to the asymptotic solution expanded by $\hbar$ gives

$$
\begin{aligned}
\widehat{u}_{B \pm}(y, \xi) & :=\mathcal{B}\left[\widehat{u}_{ \pm}\right](y, \xi) \\
& =y^{-3 / 2} 2^{1 \mp \kappa / 2} \sum_{n=0}^{+\infty} \frac{\widehat{u}_{n}^{ \pm}}{2^{n} \Gamma\left(\frac{1 \pm \kappa}{2}+n\right)}\left(\frac{2 \xi}{y^{2}} \pm \frac{1}{2}\right)^{(-1 \pm \kappa) / 2+n} .
\end{aligned}
$$

By taking the summation, it can be expressed as

$$
\widehat{u}_{B \pm}(y, \xi)=A_{ \pm} y^{-3 / 2}\left( \pm s_{ \pm}\right)^{-\gamma_{\mp}} F\left(\alpha_{ \pm}, \beta_{ \pm} ; \gamma_{ \pm} ; s_{ \pm}\right),
$$

where $F(\alpha, \beta ; \gamma ; s)$ is the Gauss hypergeometric function and

$$
\begin{aligned}
& s_{ \pm}=\left\{\begin{array}{ll}
s & \text { for } s_{+} \\
1-s & \text { for } s_{-}
\end{array} \quad \text { with } s=\frac{2 \xi}{y^{2}}+\frac{1}{2}\right. \\
& A_{ \pm}=\frac{2^{1 / 2+\gamma_{\mp}}}{\Gamma\left(\gamma_{ \pm}\right)}, \quad \alpha_{ \pm}=\frac{1}{4} \pm \frac{\kappa}{2}, \quad \beta_{ \pm}=\frac{3}{4} \pm \frac{\kappa}{2}, \quad \gamma_{ \pm}=\frac{1}{2} \pm \frac{\kappa}{2}
\end{aligned}
$$

From identities of the hypergeometric function, one finds that

$$
\begin{aligned}
F\left(\alpha_{ \pm}, \beta_{ \pm} ; \gamma_{ \pm} ; s_{ \pm}\right)= & s_{\mp}^{-\gamma_{ \pm}} \frac{\Gamma\left(\gamma_{ \pm}\right)^{2}}{\Gamma\left(\alpha_{ \pm}\right) \Gamma\left(\beta_{ \pm}\right)} F\left(1 / 4,-1 / 4 ; \gamma_{\mp} ; s_{\mp}\right) \\
& +\frac{\Gamma\left(\gamma_{ \pm}\right) \Gamma\left(-\gamma_{ \pm}\right)}{\Gamma(1 / 4) \Gamma(-1 / 4)} F\left(\alpha_{ \pm}, \beta_{ \pm} ; 1+\gamma_{ \pm} ; s_{\mp}\right) \\
= & s_{\mp}^{-\gamma_{ \pm}} \frac{\Gamma\left(\gamma_{ \pm}\right)^{2}}{2^{1-2 \alpha_{ \pm} \sqrt{\pi} \Gamma\left(2 \alpha_{ \pm}\right)}} s_{ \pm}^{\gamma_{\mp}} F\left(\alpha_{\mp}, \beta_{\mp} ; \gamma_{\mp} ; s_{\mp}\right) \\
& +\frac{\Gamma\left(\gamma_{ \pm}\right) \Gamma\left(-\gamma_{ \pm}\right)}{\Gamma(1 / 4) \Gamma(-1 / 4)} F\left(\alpha_{ \pm}, \beta_{ \pm} ; 1+\gamma_{ \pm} ; s_{\mp}\right) .
\end{aligned}
$$

\footnotetext{
${ }^{7}$ At this stage, it is useful to realize that the construction based on degenerate Weber type building blocks is intimately related to uniform-WKB approach. In uniform WKB, one starts with an ansatz $\psi(y)=\frac{1}{\sqrt{u^{\prime}(y)}} D_{\nu}\left(\frac{1}{\hbar} u(y)\right)$ where $D_{\nu}$ is parabolic cylinder (Weber) function and $\nu$ is ansatz parameter. Then, one expands $u(y)$ to a formal power series $u(y)=u_{0}(y)+\hbar u_{1}(y)+\hbar^{2} u_{2}(y)+\ldots$, see e.g. [13]. In certain sense, these two approach are very similar, both take advantage of the fact that in the $\hbar \rightarrow 0$ limit, the system would be described by harmonic minima. This is the point that actually generates the differences compared to Airy-type decomposition of Stokes graph, and makes it more suitable to obtain spectral information from the Weber type decomposition.
} 
The second term is irrelevant because it has no singularities. Thus, by picking up only the first term, one obtains

$$
\begin{aligned}
& A_{ \pm} y^{-3 / 2}\left( \pm s_{ \pm}\right)^{-\gamma_{\mp}} s_{\mp}^{-\gamma_{ \pm}} \frac{\Gamma\left(\gamma_{ \pm}\right)^{2}}{2^{1-2 \alpha_{ \pm}} \sqrt{\pi} \Gamma\left(2 \alpha_{ \pm}\right)} s_{ \pm}^{\gamma_{\mp}} F\left(\alpha_{\mp}, \beta_{\mp} ; \gamma_{\mp} ; s_{\mp}\right) \\
& =A_{\mp} y^{-3 / 2}\left(\mp s_{\mp}\right)^{-\gamma_{ \pm}} \frac{\sqrt{\pi}(-1)^{-(1+\kappa) / 2}}{\sqrt{2} \cos (\pi \kappa / 2) \Gamma\left(2 \alpha_{ \pm}\right)} F\left(\alpha_{\mp}, \beta_{\mp} ; \gamma_{\mp} ; s_{\mp}\right) \\
& =\frac{\sqrt{\pi}(-1)^{-(1+\kappa) / 2}}{\sqrt{2} \cos (\pi \kappa / 2) \Gamma\left(2 \alpha_{ \pm}\right)} \widehat{u}_{B \mp}(y, \xi) .
\end{aligned}
$$

By acting the Laplace integration and taking the Hankel contour, one obtains

$$
\begin{aligned}
\int_{C} d \xi e^{-\xi \eta} \widehat{u}_{B \pm}(y, \xi) \\
\quad=\int_{ \pm x^{2} / 4}^{+\infty} d \xi e^{-\xi \eta} \frac{\sqrt{\pi} e^{\pi i(1+\kappa) / 2}}{\sqrt{2} \cos (\pi \kappa / 2) \Gamma(1 / 2 \pm \kappa)} \widehat{u}_{B \mp}(y, \xi)\left(1-e^{-\pi i(1 \pm \kappa)}\right) \\
\quad=\int_{ \pm y^{2} / 4}^{+\infty} d \xi e^{-\xi \eta} \frac{i \sqrt{2 \pi} e^{(1 \mp 1) \pi i \kappa / 2}}{\Gamma(1 / 2 \pm \kappa)} \widehat{u}_{B \mp}(y, \xi) \quad \text { for } \quad \mathrm{IV} \rightarrow \mathrm{I} .
\end{aligned}
$$

In order to obtain the connection formula passing other Stokes curve, it is convenient to consider analytic continuation for $y$ by

$$
\begin{aligned}
& \widehat{u}_{ \pm}(y, \hbar) \stackrel{y \rightarrow e^{+\pi i / 2} y}{\longrightarrow} e^{-\pi i(1 / 2 \pm \kappa) / 2} \widehat{u}_{\mp}(y, \hbar), \\
& \widehat{u}_{ \pm}(y, \hbar) \stackrel{y \rightarrow e^{-\pi i} y}{\longrightarrow} e^{+\pi i(1 / 2 \pm \kappa)} \widehat{u}_{ \pm}(y, \hbar), \\
& \widehat{u}_{ \pm}(y, \hbar) \stackrel{y \rightarrow e^{-\pi i / 2} y}{\longrightarrow} e^{+\pi i(1 / 2 \pm \kappa) / 2} \widehat{u}_{\mp}(y, \hbar) .
\end{aligned}
$$

By repeating the similar above procedure and taking into account eq. (A.15), the connection formula for figure 7 is given by

$$
\begin{aligned}
\mathrm{IV} \rightarrow \mathrm{I}: & \Delta_{z= \pm y^{2} / 4} \widehat{\psi}_{B \pm}^{\mathrm{IV}}(y, \xi)=i \frac{\sqrt{2 \pi} e^{+(1 \mp 1) \pi i \kappa / 2}}{\Gamma(1 / 2 \pm \kappa)} \hbar^{\mp \kappa} \widehat{\psi}_{B \mp}^{\mathrm{I}}(y, \xi), \\
\mathrm{I} \rightarrow \mathrm{II}: & \Delta_{z=\mp y^{2} / 4} \widehat{\psi}_{B \mp}^{\mathrm{I}}(y, \xi)=i \frac{\sqrt{2 \pi} e^{+(1 \pm 1) \pi i \kappa / 2}}{\Gamma(1 / 2 \mp \kappa)} \hbar^{ \pm \kappa} \widehat{\psi}_{B \pm}^{\mathrm{II}}(y, \xi), \\
\mathrm{II} \rightarrow \mathrm{III}: & \Delta_{z= \pm y^{2} / 4} \widehat{\psi}_{B \pm}^{\mathrm{II}}(y, \xi)=i \frac{\sqrt{2 \pi} e^{-(3 \pm 1) \pi i \kappa / 2}}{\Gamma(1 / 2 \pm \kappa)} \hbar^{\mp \kappa} \widehat{\psi}_{B \mp}^{\mathrm{II}}(y, \xi), \\
\mathrm{III} \rightarrow \mathrm{IV}: & \Delta_{z=\mp y^{2} / 4} \widehat{\psi}_{B-}^{\mathrm{III}}(y, \xi)=i \frac{\sqrt{2 \pi} e^{-(3 \mp 1) \pi i \kappa / 2}}{\Gamma(1 / 2 \mp \kappa)} \hbar^{ \pm \kappa} \widehat{\psi}_{B \pm}^{\mathrm{IV}}(y, \xi),
\end{aligned}
$$

where $\Delta_{z}$ is the Alien derivative at $\xi=z$.

(A.29) can be viewed as the connection formula where connection formula for the Now, we are ready to obtain the global connection formula for a generic potential by using 
eq. (A.10). The result is

For figure 7 (Left) :

$$
\begin{aligned}
& \left(\begin{array}{l}
\psi_{+}^{\mathrm{IV}}(x, \hbar) \\
\psi_{-}^{\mathrm{IV}}(x, \hbar)
\end{array}\right)=\left(\begin{array}{cc}
1 & i \frac{C_{+}^{\mathrm{IV} \rightarrow \mathrm{I}}(\hbar)}{C_{-}^{\mathrm{IV} \rightarrow \mathrm{I}}(\hbar)} \frac{\sqrt{2 \pi} \hbar^{-F(\hbar)}}{\Gamma(1 / 2+F(\hbar))} \\
0 & 1
\end{array}\right)\left(\begin{array}{l}
\psi_{+}^{\mathrm{I}}(x, \hbar) \\
\psi_{-}^{\mathrm{I}}(x, \hbar)
\end{array}\right), \\
& \left(\begin{array}{l}
\psi_{+}^{\mathrm{I}}(x, \hbar) \\
\psi_{-}^{\mathrm{I}}(x, \hbar)
\end{array}\right)=\left(\begin{array}{cc}
1 & 0 \\
i \frac{C_{-}^{\mathrm{I} \rightarrow \mathrm{II}}(\hbar)}{C_{+}^{\mathrm{I} \rightarrow \mathrm{II}}(\hbar)} \frac{\sqrt{2 \pi} e^{+\pi i F(\hbar)} \hbar^{+F(\hbar)}}{\Gamma(1 / 2-F(\hbar))} & 1
\end{array}\right)\left(\begin{array}{c}
\psi_{+}^{\mathrm{II}}(x, \hbar) \\
\psi_{-}^{\mathrm{II}}(x, \hbar)
\end{array}\right), \\
& \left(\begin{array}{l}
\psi_{+}^{\mathrm{II}}(x, \hbar) \\
\psi_{-}^{\mathrm{II}}(x, \hbar)
\end{array}\right)=\left(\begin{array}{cc}
1 & i \frac{C_{+}^{\mathrm{II} \rightarrow \mathrm{III}}(\hbar)}{C_{-}^{\mathrm{II} \rightarrow \mathrm{II}}(\hbar)} \frac{\sqrt{2 \pi} e^{-2 \pi i F(\hbar)} \hbar^{-F(\hbar)}}{\Gamma(1 / 2+F(\hbar))} \\
0 & 1
\end{array}\right)\left(\begin{array}{l}
\psi_{+}^{\mathrm{III}}(x, \hbar) \\
\psi_{-}^{\mathrm{III}}(x, \hbar)
\end{array}\right), \\
& \left(\begin{array}{c}
\psi_{+}^{\mathrm{III}}(x, \hbar) \\
\psi_{-}^{\mathrm{III}}(x, \hbar)
\end{array}\right)=\left(\begin{array}{cc}
1 & 0 \\
i \frac{C_{-}^{\mathrm{III} \rightarrow \mathrm{IV}}(\hbar)}{C_{+}^{\mathrm{III} \rightarrow \mathrm{IV}}(\hbar)} \frac{\sqrt{2 \pi} e^{-\pi i F(\hbar)} \hbar^{+F(\hbar)}}{\Gamma(1 / 2-F(\hbar))} & 1
\end{array}\right)\left(\begin{array}{l}
\psi_{+}^{\mathrm{IV}}(x, \hbar) \\
\psi_{-}^{\mathrm{IV}}(x, \hbar)
\end{array}\right),
\end{aligned}
$$

For figure 7 (Right) :

$$
\begin{aligned}
\left(\begin{array}{l}
\psi_{+}^{\mathrm{IV}}(x, \hbar) \\
\psi_{-}^{\mathrm{IV}}(x, \hbar)
\end{array}\right) & =\left(\begin{array}{cc}
1 & 0 \\
i \frac{C_{-}^{\mathrm{IV} \rightarrow \mathrm{I}}(\hbar)}{C_{+}^{\mathrm{IV} \rightarrow \mathrm{I}}(\hbar)} \frac{\sqrt{2 \pi} e^{+\pi i F(\hbar)} \hbar^{+F(\hbar)}}{\Gamma(1 / 2-F(\hbar))} & 1
\end{array}\right)\left(\begin{array}{l}
\psi_{+}^{\mathrm{I}}(x, \hbar) \\
\psi_{-}^{\mathrm{I}}(x, \hbar)
\end{array}\right), \\
\left(\begin{array}{l}
\psi_{+}^{\mathrm{I}}(x, \hbar) \\
\psi_{-}^{\mathrm{I}}(x, \hbar)
\end{array}\right) & =\left(\begin{array}{cc}
1 & i \frac{C_{+}^{\mathrm{I} \rightarrow \mathrm{II}}(\hbar)}{C_{-}^{\mathrm{I} \rightarrow \mathrm{II}}(\hbar)} \frac{\sqrt{2 \pi} \hbar^{-F(\hbar)}}{\Gamma(1 / 2+F(\hbar))} \\
0 & 1
\end{array}\right)\left(\begin{array}{l}
\psi_{+}^{\mathrm{II}}(x, \hbar) \\
\psi_{-}^{\mathrm{II}}(x, \hbar)
\end{array}\right), \\
\left(\begin{array}{l}
\psi_{+}^{\mathrm{II}}(x, \hbar) \\
\psi_{-}^{\mathrm{II}}(x, \hbar)
\end{array}\right) & =\left(\begin{array}{cc}
1 & 0 \\
i \frac{C_{-}^{\mathrm{II} \rightarrow \mathrm{III}}(\hbar)}{C_{+}^{\mathrm{II} \rightarrow \mathrm{II}}(\hbar)} \frac{\sqrt{2 \pi} e^{-\pi i F(\hbar)} \hbar^{+F(\hbar)}}{\Gamma(1 / 2-F(\hbar))} & 1
\end{array}\right)\left(\begin{array}{l}
\psi_{+}^{\mathrm{III}}(x, \hbar) \\
\psi_{-}^{\mathrm{III}}(x, \hbar)
\end{array}\right), \\
\left(\begin{array}{l}
\psi_{+}^{\mathrm{III}}(x, \hbar) \\
\psi_{-}^{\mathrm{III}}(x, \hbar)
\end{array}\right) & =\left(\begin{array}{ll}
1 & i \frac{C_{+}^{\mathrm{III} \rightarrow \mathrm{IV}}(\hbar)}{C_{-}^{\mathrm{III} \rightarrow \mathrm{IV}}(\hbar)} \frac{\sqrt{2 \pi} e^{-2 \pi i F(\hbar)} \hbar^{-F(\hbar)}}{\Gamma(1 / 2+F(\hbar))} \\
0 & 1
\end{array}\right)\left(\begin{array}{l}
\psi_{+}^{\mathrm{IV}}(x, \hbar) \\
\psi_{-}^{\mathrm{IV}}(x, \hbar)
\end{array}\right),
\end{aligned}
$$

where

$$
F(\hbar)=\sum_{n=0}^{+\infty} F_{n} \hbar^{n}
$$

and $F(\hbar)$ and $C_{ \pm}^{\bullet \rightarrow \bullet}(\hbar)$ can be computed from the details of $Q(x, \hbar)$.

$F(\hbar)$ directly has the relationship with $S_{\text {odd }}(x, \hbar)$ as

$$
F(\hbar)=\mp \operatorname{Res}_{x=a_{\ell}} S_{\text {odd }}(x, \hbar),
$$

where $a_{\ell}$ is a turning point, and the sign depends on the asymptotic behavior around a turning point in the local and global coordinates. It can be shown as follows: assume that the asymptotic behavior around a turning point in the local and global Stokes graphs is 
the left panel in figure 7 . Since

$$
\widehat{S}_{\text {odd }}(y, \hbar)=\hbar^{-1} \frac{y}{2}-\frac{\kappa}{y}+O\left(y^{-3}\right),
$$

one finds that

$$
\operatorname{Res}_{y=0} \widehat{S}_{\text {odd }}(y, \hbar)=-\kappa \text {. }
$$

Suppose a neighborhood around $x=a_{\ell}$ where $y\left(a_{\ell}, \hbar\right)=0$. From eq. (A.3),

$$
\operatorname{Res}_{y=0} \widehat{S}_{\text {odd }}(y, \hbar)=\operatorname{Res}_{x=a_{\ell}} S_{\text {odd }}(x, \hbar),
$$

which gives the translation of $\kappa$ in the local coordinate to $F(\hbar)$ defined in the global coordinate. By comparing the local connection formula with the global one, eq. (A.42) with the minus sign can be obtained. By repeating the same procedure, the relatinship of $F(\hbar)$ and $S_{\text {odd }}(x, \hbar)$ when the asymptotic behavour around a turning point in the local and/or global coordinates change can be obtained. As a result, $F(\hbar)=-\operatorname{Res}_{x=a_{\ell}} S_{\text {odd }}(x, \hbar)$ when the asymptotic behavior around a turning point in the local and global coordinate matches and $F(\hbar)=+\operatorname{Res}_{x=a_{\ell}} S_{\text {odd }}(x, \hbar)$ if it does not.

In order to obtain $C_{ \pm}(\hbar)$, we use eqs. (A.1), (A.3), and (A.10). From eq. (A.1) with a fixed order of $\hbar$, one finds

$$
\begin{aligned}
& \frac{y_{0}(x)}{2} \frac{d y_{0}(x)}{d x}= \pm \sqrt{Q_{0}(x)}= \pm S_{\text {odd },-1}(x), \\
& \frac{y_{0}(x)^{2}}{2} \frac{d y_{0}(x)}{d x} \frac{d y_{1}(x)}{d x}+\left(\frac{d y_{0}(x)}{d x}\right)^{2} \frac{y_{0}(x) y_{1}(x)}{2}-\left(\frac{d y_{0}(x)}{d x}\right)^{2} \kappa=Q_{1}(x),
\end{aligned}
$$

and recursionally solving them with $y\left(a_{\ell}, \hbar\right)=0$ where $a_{\ell}$ is a turning point gives the coordinate transformation. Notice that the sign $+/-$ in the first line of eq. (A.46) corresponds to the left/right panel in figure 7. Furthermore, by combining with (A.3) and (A.10), one has

$$
\begin{aligned}
C_{ \pm}(\hbar) y^{\mp \kappa} \exp \left[ \pm \frac{1}{\hbar} \frac{y^{2}}{4} \pm \int_{\infty}^{y} d y\left(\widehat{S}_{\text {odd }}(y, \hbar)-\frac{1}{\hbar} \widehat{S}_{\text {odd },-1}(y)+\frac{\kappa}{y}\right)\right] \\
\quad= \begin{cases}\exp \left[ \pm \frac{1}{\hbar} \int_{a_{\ell}}^{x} d x \sqrt{Q_{0}(x)} \pm \int_{\infty}^{x} d x\left(S_{\text {odd }}(x, \hbar)-\frac{1}{\hbar} S_{\text {odd },-1}(x)\right)\right] & \text { for }+\sqrt{Q_{0}(x)} \\
\exp \left[\mp \frac{1}{\hbar} \int_{a_{\ell}}^{x} d x \sqrt{Q_{0}(x)} \mp \int_{\infty}^{x} d x\left(S_{\text {odd }}(x, \hbar)-\frac{1}{\hbar} S_{\text {odd },-1}(x)\right)\right] & \text { for }-\sqrt{Q_{0}(x)}\end{cases}
\end{aligned}
$$

and recursively solve it for $C_{ \pm}(\hbar)$ order by order after obtaining the coordinate transformation from eq. (A.46). Here, we focus on $a_{\ell}=0$. From eq. (A.46), the 0th and 1st orders of $y$ are obtained as

$$
\begin{aligned}
& y_{0}(x)=\left\{\begin{array}{ll}
\sqrt{2} N^{1 / 2} x-\frac{1}{48 \sqrt{2}} N^{5 / 2} x^{3}+O\left(x^{5}\right) & \text { for }+\sqrt{Q_{0}(x)} \\
\sqrt{2} i N^{1 / 2} x-\frac{i}{48 \sqrt{2}} N^{5 / 2} x^{3}+O\left(x^{5}\right) & \text { for }-\sqrt{Q_{0}(x)}
\end{array},\right. \\
& y_{1}(x)=\left\{\begin{array}{ll}
-\frac{1}{16 \sqrt{2}} E N^{1 / 2} x-\frac{3}{2048 \sqrt{2}} E N^{5 / 2} x^{3}+O\left(x^{5}\right) & \text { for }+\sqrt{Q_{0}(x)} \\
-\frac{i}{16 \sqrt{2}} E N^{1 / 2} x-\frac{3 i}{2048 \sqrt{2}} E N^{5 / 2} x^{3}+O\left(x^{5}\right) & \text { for }-\sqrt{Q_{0}(x)}
\end{array},\right.
\end{aligned}
$$


where $\kappa$ should be chosen as $\kappa \sim F_{0}= \pm E / N$ to cancel divergence. From these results the leading order of $C_{ \pm}(\hbar)$ is given from eq. (A.47) by ${ }^{8}$

$$
C_{ \pm, 0}=\left\{\begin{array}{ll}
\lim _{x \rightarrow 0} \frac{\exp \left[ \pm \int_{\infty}^{x} S_{\text {odd }, 0}(x)\right]}{y_{0}(x)^{\mp \kappa}}=\exp \left[ \pm \frac{\pi i E}{2 N}\right]\left(\frac{32}{N}\right)^{ \pm \frac{E}{2 N}} & \text { for }+\sqrt{Q_{0}(x)} \\
\lim _{x \rightarrow 0} \frac{\exp \left[\mp \int_{\infty}^{x} S_{\text {odd }, 0}(x)\right]}{y_{0}(x)^{\mp \kappa}}=\left(\frac{32}{N}\right)^{\mp \frac{E}{2 N}} & \text { for }-\sqrt{Q_{0}(x)}
\end{array} .\right.
$$

Finally, we define the normalization and branchcut matrices as

$$
\begin{aligned}
\mathcal{N}_{a_{1}, a_{2}} & =\left(\begin{array}{cc}
e^{+\frac{1}{\hbar} \int_{a_{1}}^{a_{2}} d x S_{\mathrm{odd},-1}(x)} & 0 \\
0 & e^{-\frac{1}{\hbar} \int_{a_{1}}^{a_{2}} d x S_{\mathrm{odd},-1}(x)}
\end{array}\right), \\
\mathcal{T} & =\left(\begin{array}{cc}
0 & -i \\
-i & 0
\end{array}\right),
\end{aligned}
$$

where it has only $S_{\text {odd, }-1}(x)$ in the normalization matrix because the wavefunction is normalized at $x=\infty$ for $S_{\text {odd, } n>-1}(x)$, and the branchcut matrix can be defined by $S_{\text {odd }}(x, \hbar) \rightarrow-S_{\text {odd }}(x, \hbar)$ in the wavefunction.

\section{A.2 Construction of the dictionary}

We now construct the dictionary translating cycles between the Airy-type and the DW-type building blocks of the Stokes graph.

\section{A.2.1 A-cycle}

For $Q(x, \hbar)=2[1-\cos (N x)-E]$, the $A$-cycle around $x=0$ for the Airy-type is given by

$$
A=e^{\oint_{a_{1}}^{a_{2}} d x S_{\text {odd }}(x, \hbar)}
$$

where $a_{1,2}$ is the turning point given by $a_{2,1}= \pm \frac{\arccos (1-E)}{N}$ with $0<E<2$. For simplicity, we suppose that a branch-cut connecting with both $a_{1}$ and $a_{2}$ as end points exists. When replacing $E \rightarrow E \hbar$ to obtain the $A$-cycle for the DW-type, the turning points collide with each other at $x=0$. By denoting $S_{\text {odd }}^{\mathrm{DW}}(x, \hbar)$ instead of $S_{\text {odd }}(x, \hbar)$ for the DW-type, which is calculated by $Q(x, \hbar)=2[1-\cos (N x)-E \hbar]$, the $\mathcal{A}$-cycle is defined as

$$
\mathcal{A}=e^{\oint_{|x| \ll 1} d x S_{\text {odd }}^{\text {DW }}(x, \hbar)}=: e^{\mp 2 \pi i F(\hbar)},
$$

where $F(\hbar)$ is given by eq. (A.42), and the sign depends on the asymptotic behavior around a turning point in the global coordinate.

\footnotetext{
${ }^{8}$ In this paper, we took the asymptotic behavior around turning points as the right panel in figure 7 . If one takes the left panel, the identification of $B$-cycle in eq. (A.60) gives a slightly different form. However, by taking into account the difference of $C_{ \pm}(\hbar)$, the final result is unchanged.
} 

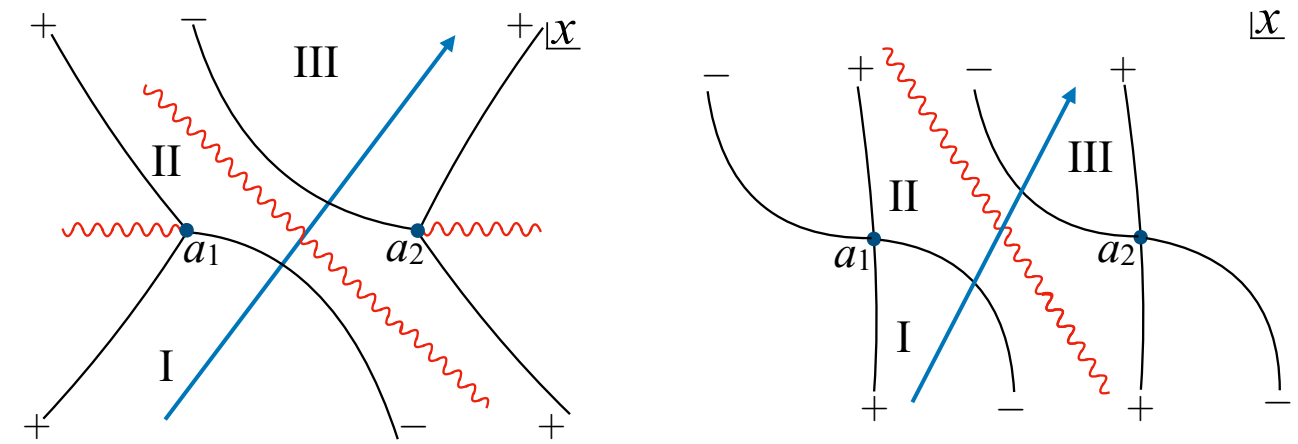

Figure 9. The orbit we consider to compare the connection formula from Airy-type (left) and the DW-type (right).

\section{A.2.2 $B$-cycle}

We consider the orbit in figure 9 and compare the monodromy matrix obtained by connection formula from the Airy-type and the DW-type. Those are calculated by

$$
\begin{aligned}
& \text { Airy : } \psi_{\mathrm{I}}=M_{-} N_{a_{1}, a_{2}} T M_{-}^{-1} N_{a_{2}, a_{1}} \psi_{\mathrm{III}}=: D \psi_{\mathrm{III}}, \\
& \text { DW : } \psi_{\mathrm{I}}=\mathcal{M}_{1 \ominus}^{\mathrm{IV} \rightarrow \mathrm{I}} \mathcal{N}_{a_{1}, a_{2}} \mathcal{T} \mathcal{M}_{2 \ominus}^{\mathrm{II} \rightarrow \mathrm{III}} \mathcal{N}_{a_{2}, a_{1}} \psi_{\mathrm{III}}=: \mathcal{D} \psi_{\mathrm{III}} .
\end{aligned}
$$

Notice that $\psi_{\mathrm{I}}^{+}=0$ due to the asymptotic behaviour, thus $D_{21}$ and $\mathcal{D}_{21}$ are comparable with each other. Those are obtained as

$$
\begin{array}{ll}
\text { Airy }: & D_{21}=-i(1+B), \\
\text { DW }: & \mathcal{D}_{21}=-i(1+\mathcal{B}),
\end{array}
$$

where

$$
\begin{aligned}
& B=e^{2 \int_{a_{1}}^{a_{2}} d x S_{\text {odd }}(x, \hbar)}, \\
& \mathcal{B}=2 \pi \mathcal{B}_{0} \prod_{\ell=1}^{2} \frac{C_{\ell-}(\hbar)}{C_{\ell+}(\hbar)} \frac{e^{(-1)^{\ell+1} \pi i F_{\ell}(\hbar)} \hbar^{F_{\ell}(\hbar)}}{\Gamma\left(1 / 2-F_{\ell}(\hbar)\right)},
\end{aligned}
$$

with $\mathcal{B}_{0}=e^{\frac{2}{\hbar} \int_{a_{1}}^{a_{2}} d x S_{\text {odd },-1}^{\mathrm{DW}}(x)}$, where $\ell$ is the label of turning points.

Open Access. This article is distributed under the terms of the Creative Commons Attribution License (CC-BY 4.0), which permits any use, distribution and reproduction in any medium, provided the original author(s) and source are credited.

\section{References}

[1] J. Ecalle, Les Fonctions Resurgentes, Vol. I-III, Publ. Math. Orsay, (1981).

[2] E. Brézin, J.C. Le Guillou and J. Zinn-Justin, Perturbation Theory at Large Order. 2. Role of the Vacuum Instability, Phys. Rev. D 15 (1977) 1558 [InSPIRE].

[3] L.N. Lipatov, Divergence of the Perturbation Theory Series and the Quasiclassical Theory, Sov. Phys. JETP 45 (1977) 216 [Zh. Eksp. Teor. Fiz. 72 (1977) 411] [INSPIRE]. 
[4] G. Alvarez and C. Casares, Exponentially small corrections in the asymptotic expansion of the eigenvalues of the cubic anharmonic oscillator, J. Phys. A 33 (2000) 5171.

[5] G. Alvarez and C. Casares, Uniform asymptotic and JWKB expansions for anharmonic oscillators, J. Phys. A 33 (2000) 2499.

[6] G. Alvarez, Langer-Cherry derivation of the multi-instanton expansion for the symmetric double well, J. Math. Phys. 45 (2004) 3095.

[7] J. Zinn-Justin and U.D. Jentschura, Multi-instantons and exact results I: Conjectures, WKB expansions, and instanton interactions, Annals Phys. 313 (2004) 197 [quant-ph/0501136] [INSPIRE].

[8] J. Zinn-Justin and U.D. Jentschura, Multi-instantons and exact results II: Specific cases, higher-order effects, and numerical calculations, Annals Phys. 313 (2004) 269 [quant-ph/0501137] [INSPIRE].

[9] U.D. Jentschura, A. Surzhykov and J. Zinn-Justin, Multi-instantons and exact results. III: Unification of even and odd anharmonic oscillators, Annals Phys. 325 (2010) 1135 [arXiv:1001.3910] [INSPIRE].

[10] U.D. Jentschura and J. Zinn-Justin, Multi-instantons and exact results. IV: Path integral formalism, Annals Phys. 326 (2011) 2186 [InSPIRE].

[11] G.V. Dunne and M. Ünsal, Generating nonperturbative physics from perturbation theory, Phys. Rev. D 89 (2014) 041701 [arXiv:1306.4405] [InSPIRE].

[12] G. Basar, G.V. Dunne and M. Ünsal, Resurgence theory, ghost-instantons, and analytic continuation of path integrals, JHEP 10 (2013) 041 [arXiv:1308.1108] [INSPIRE].

[13] G.V. Dunne and M. Ünsal, Uniform WKB, Multi-instantons, and Resurgent Trans-Series, Phys. Rev. D 89 (2014) 105009 [arXiv:1401.5202] [InSPIRE].

[14] T. Misumi, M. Nitta and N. Sakai, Resurgence in sine-Gordon quantum mechanics: Exact agreement between multi-instantons and uniform WKB, JHEP 09 (2015) 157 [arXiv: 1507.00408] [INSPIRE].

[15] A. Behtash, G.V. Dunne, T. Schäfer, T. Sulejmanpasic and M. Ünsal, Toward Picard-Lefschetz theory of path integrals, complex saddles and resurgence, Ann. Math. Sci. Appl. 02 (2017) 95 [arXiv:1510.03435] [INSPIRE].

[16] I. Gahramanov and K. Tezgin, Remark on the Dunne-Ünsal relation in exact semiclassics, Phys. Rev. D 93 (2016) 065037 [arXiv:1512.08466] [InSPIRE].

[17] G.V. Dunne and M. Ünsal, WKB and Resurgence in the Mathieu Equation, arXiv: 1603.04924 [INSPIRE].

[18] T. Fujimori, S. Kamata, T. Misumi, M. Nitta and N. Sakai, Nonperturbative contributions from complexified solutions in $\mathbb{C} P^{N-1}$ models, Phys. Rev. D 94 (2016) 105002 [arXiv: 1607.04205] [INSPIRE].

[19] G. Basar, G.V. Dunne and M. Ünsal, Quantum Geometry of Resurgent Perturbative/Nonperturbative Relations, JHEP 05 (2017) 087 [arXiv:1701.06572] [INSPIRE].

[20] T. Fujimori, S. Kamata, T. Misumi, M. Nitta and N. Sakai, Exact resurgent trans-series and multibion contributions to all orders, Phys. Rev. D 95 (2017) 105001 [arXiv:1702.00589] [INSPIRE]. 
[21] N. Sueishi, 1/є problem in resurgence, PTEP 2021 (2021) 013B01 [arXiv:1912.03518] [INSPIRE].

[22] M. Mariño, Open string amplitudes and large order behavior in topological string theory, JHEP 03 (2008) 060 [hep-th/0612127] [INSPIRE].

[23] M. Mariño, R. Schiappa and M. Weiss, Nonperturbative Effects and the Large-Order Behavior of Matrix Models and Topological Strings, Commun. Num. Theor. Phys. 2 (2008) 349 [arXiv: 0711.1954] [INSPIRE].

[24] S. Pasquetti and R. Schiappa, Borel and Stokes Nonperturbative Phenomena in Topological String Theory and c=1 Matrix Models, Annales Henri Poincaré 11 (2010) 351 [arXiv: 0907.4082] [INSPIRE].

[25] S. Garoufalidis, A. Its, A. Kapaev and M. Mariño, Asymptotics of the instantons of Painlevé I, Int. Math. Res. Not. 2012 (2012) 561 [arXiv: 1002.3634] [INSPIRE].

[26] I. Aniceto, R. Schiappa and M. Vonk, The Resurgence of Instantons in String Theory, Commun. Num. Theor. Phys. 6 (2012) 339 [arXiv:1106.5922] [INSPIRE].

[27] M. Mariño, Lectures on non-perturbative effects in large $N$ gauge theories, matrix models and strings, Fortsch. Phys. 62 (2014) 455 [arXiv:1206.6272] [INSPIRE].

[28] I. Aniceto and R. Schiappa, Nonperturbative Ambiguities and the Reality of Resurgent Transseries, Commun. Math. Phys. 335 (2015) 183 [arXiv:1308.1115] [INSPIRE].

[29] A. Grassi, Y. Hatsuda and M. Mariño, Quantization conditions and functional equations in ABJ $(M)$ theories, J. Phys. A 49 (2016) 115401 [arXiv:1410.7658] [inSPIRE].

[30] R. Couso-Santamaría, R. Schiappa and R. Vaz, Finite $N$ from Resurgent Large $N$, Annals Phys. 356 (2015) 1 [arXiv:1501.01007] [InSPIRE].

[31] Y. Hatsuda and M. Mariño, Exact quantization conditions for the relativistic Toda lattice, JHEP 05 (2016) 133 [arXiv:1511.02860] [INSPIRE].

[32] S. Franco, Y. Hatsuda and M. Mariño, Exact quantization conditions for cluster integrable systems, J. Stat. Mech. 1606 (2016) 063107 [arXiv: 1512.03061] [INSPIRE].

[33] R. Couso-Santamaría, R. Schiappa and R. Vaz, On asymptotics and resurgent structures of enumerative Gromov-Witten invariants, Commun. Num. Theor. Phys. 11 (2017) 707 [arXiv: 1605. 07473] [INSPIRE].

[34] R. Couso-Santamaría, M. Mariño and R. Schiappa, Resurgence Matches Quantization, J. Phys. A 50 (2017) 145402 [arXiv: 1610.06782] [INSPIRE].

[35] G.V. Dunne and M. Ünsal, Resurgence and Trans-series in Quantum Field Theory: The $C P(N-1)$ Model, JHEP 11 (2012) 170 [arXiv:1210.2423] [INSPIRE].

[36] A. Cherman, D. Dorigoni, G.V. Dunne and M. Ünsal, Resurgence in Quantum Field Theory: Nonperturbative Effects in the Principal Chiral Model, Phys. Rev. Lett. 112 (2014) 021601 [arXiv: 1308.0127] [INSPIRE].

[37] A. Cherman, D. Dorigoni and M. Ünsal, Decoding perturbation theory using resurgence: Stokes phenomena, new saddle points and Lefschetz thimbles, JHEP 10 (2015) 056 [arXiv: 1403.1277] [INSPIRE].

[38] T. Misumi, M. Nitta and N. Sakai, Neutral bions in the $\mathbb{C} P^{N-1}$ model, JHEP 06 (2014) 164 [arXiv: 1404.7225] [INSPIRE]. 
[39] T. Misumi, M. Nitta and N. Sakai, Neutral bions in the $\mathbb{C} P^{N-1}$ model for resurgence, J. Phys. Conf. Ser. 597 (2015) 012060 [arXiv: 1412.0861] [inSPIRE].

[40] T. Sulejmanpasic, Global Symmetries, Volume Independence, and Continuity in Quantum Field Theories, Phys. Rev. Lett. 118 (2017) 011601 [arXiv:1610.04009] [InSPIRE].

[41] G.V. Dunne and M. Ünsal, What is QFT? Resurgent trans-series, Lefschetz thimbles, and new exact saddles, PoS LATTICE2015 (2016) 010 [arXiv:1511.05977] [InSPIRE].

[42] P.V. Buividovich, G.V. Dunne and S.N. Valgushev, Complex Path Integrals and Saddles in Two-Dimensional Gauge Theory, Phys. Rev. Lett. 116 (2016) 132001 [arXiv:1512.09021] [INSPIRE].

[43] S. Gukov, M. Mariño and P. Putrov, Resurgence in complex Chern-Simons theory, arXiv: 1605.07615 [INSPIRE].

[44] E. Delabaere, H. Dillinger and F. Pham, Exact semiclassical expansions for one-dimensional quantum oscillators, J. Math. Phys. 38 (1997) 6126.

[45] E. Delabaere and F. Pham, Resurgent methods in semiclassical asymptotics, Ann. Inst. H. Poincare 71 (1999) 1.

[46] Y. Takei, An explicit description of the connection formula for the first Painleve equation, Toward the Exact WKB Analysis of Differential Equations, Linear or Non-Linear, Kyoto University Press, (2000), pp. 271-296.

[47] Y. Takei, Sato's conjecture for the Weber equation and transformation theory for Schrödinger equations with a merging pair of turning points, RIMS Kokyuroku Bessatsu B 10 (2008) 205.

[48] Y. Takei, On the connection formula for the first Painleve equation: from the viewpoint of the exact WKB analysis(Painleve Transcendents and Asymptotic Analysis), Kyoto University Research Information Repository 931 (1995) 70.

[49] T. Kawai and Y. Takei Algebraic Analysis of Singular Perturbation Theory, American Mathematical Society, Providence, R.I., U.S.A. (2005).

[50] T. Aoki, T. Kawai and Y. Takei, The Bender-Wu analysis and the Voros theory. II, Adv. Stud. Pure Math 54 (2009) 19.

[51] A. Fruchard and R. Schäfke, On the parametric resurgence for a certain singularly perturbed linear differential equation of second order, in Asymptotics in Dynamics, Geometry and PDEs; Generalized Borel Summation, vol. II, Edizioni della Normale (2010), pp. 213-243.

[52] K. Iwaki and T. Nakanishi, Exact WKB analysis and cluster algebras, J. Phys. A 47 (2014) 474009 [arXiv: 1401.7094 ].

[53] A.-K. Kashani-Poor and J. Troost, Pure $\mathcal{N}=2$ super Yang-Mills and exact WKB, JHEP 08 (2015) 160 [arXiv: 1504.08324] [InSPIRE].

[54] A.-K. Kashani-Poor, Quantization condition from exact WKB for difference equations, JHEP 06 (2016) 180 [arXiv:1604.01690] [INSPIRE].

[55] S.K. Ashok, D.P. Jatkar, R.R. John, M. Raman and J. Troost, Exact WKB analysis of $\mathcal{N}=2$ gauge theories, JHEP 07 (2016) 115 [arXiv: 1604.05520] [INSPIRE].

[56] K. Ito, M. Mariño and H. Shu, TBA equations and resurgent Quantum Mechanics, JHEP 01 (2019) 228 [arXiv: 1811.04812] [INSPIRE].

[57] L. Hollands and A. Neitzke, Exact WKB and abelianization for the $T_{3}$ equation, Commun. Math. Phys. 380 (2020) 131 [arXiv:1906.04271] [INSPIRE]. 
[58] S.K. Ashok, P.N. Bala Subramanian, A. Bawane, D. Jain, D.P. Jatkar and A. Manna, Exact WKB Analysis of $\mathbb{C P}^{1}$ Holomorphic Blocks, JHEP 10 (2019) 075 [arXiv:1907.05031] [INSPIRE].

[59] K. Ito and H. Shu, TBA equations for the Schrödinger equation with a regular singularity, $J$. Phys. A 53 (2020) 33 [arXiv:1910.09406] [INSPIRE].

[60] K. Imaizumi, Exact WKB analysis and TBA equations for the Mathieu equation, Phys. Lett. $B 806$ (2020) 135500 [arXiv:2002.06829] [INSPIRE].

[61] I. Coman, P. Longhi and J. Teschner, From quantum curves to topological string partition functions II, arXiv:2004.04585 [INSPIRE].

[62] D.G.L. Allegretti, On the wall-crossing formula for quadratic differentials, arXiv:2006.08059 [INSPIRE].

[63] T. Kuwagaki, Sheaf quantization from exact WKB analysis, arXiv:2006.14872 [INSPIRE].

[64] N. Sueishi, S. Kamata, T. Misumi and M. Ünsal, On exact-WKB analysis, resurgent structure, and quantization conditions, JHEP 12 (2020) 114 [arXiv:2008.00379] [INSPIRE].

[65] Y. Emery, TBA Equations and Quantization Conditions, arXiv:2008.13680 [INSPIRE].

[66] S. Enomoto and T. Matsuda, The exact WKB for cosmological particle production, JHEP 03 (2021) 090 [arXiv: 2010.14835] [INSPIRE].

[67] H. Taya, T. Fujimori, T. Misumi, M. Nitta and N. Sakai, Exact WKB analysis of the vacuum pair production by time-dependent electric fields, JHEP 03 (2021) 082 [arXiv:2010.16080] [INSPIRE].

[68] F. Yan, Exact WKB and the quantum Seiberg-Witten curve for $4 d N=2$ pure $\mathrm{SU}(3)$ Yang-Mills, Part I: Abelianization, arXiv:2012.15658 [INSPIRE].

[69] D. Gaiotto, A. Kapustin, Z. Komargodski and N. Seiberg, Theta, Time Reversal, and Temperature, JHEP 05 (2017) 091 [arXiv: 1703.00501] [INSPIRE].

[70] M. Ünsal, Strongly coupled QFT dynamics via TQFT coupling, arXiv:2007.03880 [INSPIRE].

[71] M. Ünsal and L.G. Yaffe, Center-stabilized Yang-Mills theory: Confinement and large N volume independence, Phys. Rev. D 78 (2008) 065035 [arXiv:0803.0344] [INSPIRE].

[72] M. Ünsal, Abelian duality, confinement, and chiral symmetry breaking in QCD(adj), Phys. Rev. Lett. 100 (2008) 032005 [arXiv:0708.1772] [InSPIRE].

[73] M. Ünsal, Magnetic bion condensation: A New mechanism of confinement and mass gap in four dimensions, Phys. Rev. D 80 (2009) 065001 [arXiv:0709.3269] [INSPIRE].

[74] M. Shifman and M. Ünsal, QCD-like Theories on $R_{3} \times S_{1}$ : A Smooth Journey from Small to Large $r\left(S_{1}\right)$ with Double-Trace Deformations, Phys. Rev. D 78 (2008) 065004 [arXiv:0802.1232] [INSPIRE].

[75] E. Poppitz and M. Ünsal, Conformality or confinement: (IR)relevance of topological excitations, JHEP 09 (2009) 050 [arXiv: 0906.5156] [INSPIRE].

[76] M.M. Anber and E. Poppitz, Microscopic Structure of Magnetic Bions, JHEP 06 (2011) 136 [arXiv: 1105.0940] [INSPIRE].

[77] E. Poppitz, T. Schäfer and M. Ünsal, Continuity, Deconfinement, and (Super) Yang-Mills Theory, JHEP 10 (2012) 115 [arXiv: 1205. 0290] [INSPIRE]. 
[78] T. Misumi and T. Kanazawa, Adjoint $Q C D$ on $\mathbb{R}^{3} \times S^{1}$ with twisted fermionic boundary conditions, JHEP 06 (2014) 181 [arXiv: 1405.3113] [INSPIRE].

[79] T. Fujimori, E. Itou, T. Misumi, M. Nitta and N. Sakai, Confinement-deconfinement crossover in the lattice $\mathbb{C} P^{N-1}$ model, Phys. Rev. D 100 (2019) 094506 [arXiv:1907.06925] [INSPIRE].

[80] T. Misumi, T. Fujimori, E. Itou, M. Nitta and N. Sakai, Lattice study on the twisted $\mathbb{C} P^{N-1}$ models on $\mathbb{R} \times S^{1}$, PoS LATTICE2019 (2019) 015 [arXiv:1911.07398] [INSPIRE].

[81] T. Fujimori, E. Itou, T. Misumi, M. Nitta and N. Sakai, Lattice $\mathbb{C} P^{N-1}$ model with $\mathbb{Z}_{N}$ twisted boundary condition: bions, adiabatic continuity and pseudo-entropy, JHEP 08 (2020) 011 [arXiv: 2006. 05106] [INSPIRE].

[82] M.C. Gutzwiller, Periodic orbits and classical quantization conditions, J. Math. Phys. 12 (1971) 343 [INSPIRE].

[83] M. Ünsal, Theta dependence, sign problems and topological interference, Phys. Rev. D 86 (2012) 105012 [arXiv:1201.6426] [INSPIRE].

[84] Y. Kikuchi and Y. Tanizaki, Global inconsistency, 't Hooft anomaly, and level crossing in quantum mechanics, PTEP 2017 (2017) 113B05 [arXiv:1708.01962] [INSPIRE].

[85] S. Gukov and A. Kapustin, Topological Quantum Field Theory, Nonlocal Operators, and Gapped Phases of Gauge Theories, arXiv:1307.4793 [INSPIRE].

[86] A. Kapustin and N. Seiberg, Coupling a QFT to a TQFT and Duality, JHEP 04 (2014) 001 [arXiv: 1401.0740] [INSPIRE].

[87] R. Rajaraman, Solitons And Instantons. An Introduction To Solitons And Instantons In Quantum Field Theory, North-holland, Amsterdam, Netherlands, (1982). 